Revised for publication in Powder Technology (POWTEC-D-16-01079)

\title{
Pre-Rolling Leaning of Microparticles
}

\author{
Bahman Farzi, Chaitanya Krishna Prasad Vallabh, James D. Stephens, and Cetin Cetinkaya* \\ Department of Mechanical and Aeronautical Engineering \\ Photo-Acoustics Research Laboratory \\ Center for Advanced Materials Processing \\ Clarkson University \\ Potsdam, New York 13699-5725, USA
}

\section{Version 03.28}

January 4, 2017

*Author to whom correspondence should be addressed.

| Email: cetin@clarkson.edu | Phone: (315) 268-6514 | Fax: (315) 268-6695 | 


\section{ABSTRACT}

At nano/micro-scales, the resistance of a particle to rolling is a critical factor in many applications and biological phenomena as it affects particle adhesion, motion and flow as well as particle manipulation (e.g. picking and placing of microparticles). In present work, a non-contact and a noninvasive experimental method is detailed and applied to determine the pre-rolling critical leaning angle (CLA) of single microparticles. Transient Rayleigh surface acoustic waves (SAW) are utilized as the excitation mechanism and interferometry and image processing as detection/monitoring and analysis techniques for capturing the micro/nano-scale dynamics of the microparticles. The CLA values for a set of 30 PSL (polystyrene latex) microparticles with a diameter of $14.9 \pm 0.6 \mu \mathrm{m}$ on a soda-lime glass substrate are reported. The CLA of the studied particles are determined to be between 0.9 to $7.8^{\circ}$. It is also observed that during a SAW field burst cycle, microparticles could change their drifting (rolling and/or sliding) directions, speeds and accelerations. The trajectory of a particle is often found to be non-linear. This nonlinear behavior is attributed to the inhomogeneity of the surface properties of the particles and the substrate as well as possible electrical charge density, chemistry variations and potential contamination on surfaces. Moreover, the effect of electric charge (developed due to the triboelectric effect) on the particle drifting motion is investigated. It is found that the percentage of drifting particles is not only a function of the amplitude of the excitation field but also a function of possible electrostatic charges developed on the particles due to the drifting motion on the substrate. In addition to their potential uses in particle manipulation, removal and adhesion characterization, the reported results could be utilized in numerical simulations of microparticle motion and deposition. 
Keywords: microparticle rolling; microparticle adhesion; critical leaning angle; non-contact adhesion characterization. 


\section{Graphical Abstract}

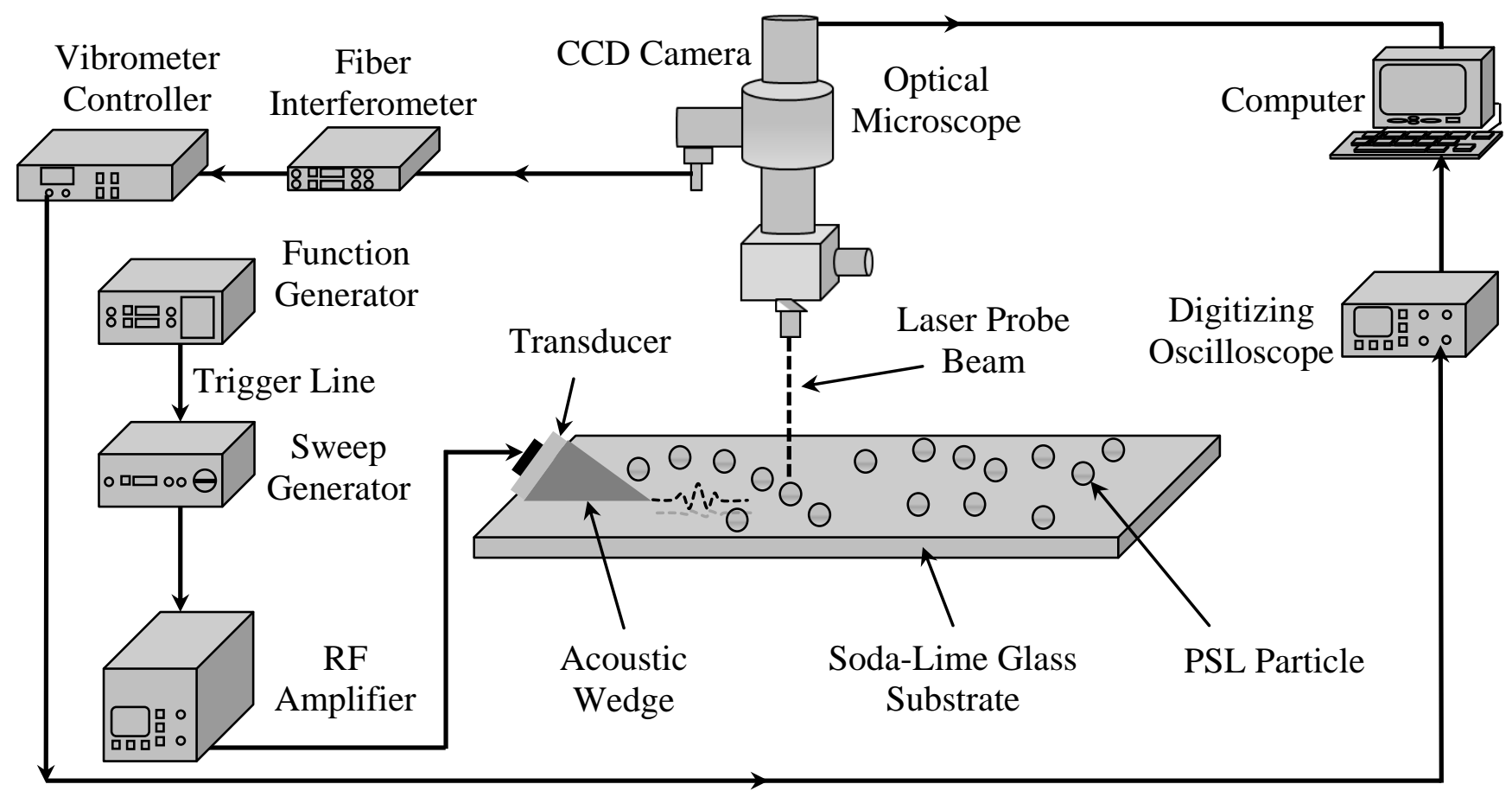




\section{Highlights}

- An experimental technique for determining the critical leaning angle of a microparticle.

- Currently there exists no analytical expression for predicting critical leaning angles.

- The presented measurement approach is based on surface acoustic wave excitation.

- The key advantage of the current approach is its non-contact and non-invasive nature. 


\section{INTRODUCTION}

In nano/micro-scale dynamics, motion begins with the dislodgement of a particle from its contact zone. In microparticle detachment and removal, rolling requires significantly less force/effort than an out-of-plane lift-off removal mechanism. Understanding the dynamics of the particle before and during the rolling is crucial in many applications, as microparticle adhesion and dynamics is key in a wide spectrum of industrial processes (semiconductor manufacturing [1], and the adhesion of toner/ink particles in printing/copying [2]), and biological phenomena (cell targeting, cell sorting, drug delivery, and cell rolling in leukocyte extravasation and metastatic cancer $[3,4])$.

One of the major factors involving the rolling of the micro-objects is the rolling resistance of microparticles and their critical leaning angle (CLA) prior to the initiation of free-rolling and/or complete detachment. Development of accurate/realistic modeling of adhesion and CLA determination of microparticles has the potential to enhance the accuracy of computational simulations of such systems with techniques such as discrete element methods (DEM), particlescale finite element (FE), and computational fluid dynamics (CFD) approaches.

An elastic particle in contact with a dry flat elastic substrate induces short-range forces (van der Waals force) and elastic/strain forces between the particle and the substrate, leading to restitutive deformation of the particle and the substrate at the point of contact [5]. In out-of-plane displacement, the force-displacement relationship for soft elastic particles on a stiff elastic half space is now known as the JKR (Johnson Kendall and Roberts) adhesion model [6], and the approach is later generalized for material pairs with broader mechanical properties [7]. Adhesion is a particle-diameter proportional force. As a result, it becomes particularly significant at nano/micro- 
meter-scale. As the scale shrinks to the micrometer-scale, adhesion often dominates the volumeand surface-proportional forces (e.g. weight and electrical charge) since adhesion is proportional to particle diameter (according to the JKR adhesion model). Similar to the out-of-plane (onedimensional) adhesion mechanics, when an external lateral force (or rolling moment) is applied on the particle, a restitutive moment at the contact point is induced, leading to a two-degree of freedom (planar) motion. While the bond is intact, the asymmetric pressure distribution at the contact zone results in an elastic restitutive moment causing the particle to undergo free rotational oscillations with respect to its equilibrium point. Above a critical value of leaning, under lateral loading, the restitutive moment is unable to restore the stable position of the particle, resulting in free-rolling and/or sliding of the particle on the substrate. Rolling motion involves the change of contact area at the leading edge and at the trailing edge of the contact with the surface, resulting in an asymmetry in the pressure distribution at the contact zone. During the particle rolling, the leading edge of the contact area establishes new contact as peeling off the trailing edge takes place.

The moment balance of forces acting on a particle in obtaining a detachment criterion has been traditionally used for predicting the onset of rolling-based micro/nano-particle detachment. This simple criterion assumes neither the build-up of a resisting moment nor a critical leaning angle prior to free-rolling of the particle. However, it has been demonstrated both analytically [8] and experimentally [9] that the adhesion bond between a microparticle and a surface indeed creates a resistance against rolling initiation. Similarly, utilizing a custom-made nano-manipulator implemented in the vacuum chamber of a scanning electron microscope (SEM), critical leaning angle and rolling resistance moment-based microparticle adhesion characterization technique was 
reported [10]. This type of rolling resistance and corresponding CLA is analogous to the static friction force observed prior to sliding in linear friction experiments under increased lateral force.

Currently there exists no analytical expression for predicting CLA while the rolling stiffness constant of this initial elastic deformation/leaning is predicted analytically from the elastic and adhesion properties of the materials involved [8]. Later, the rolling resistance effect was demonstrated experimentally by interferometrically detecting resonance frequencies of rocking microparticles under acoustic base excitation [5,9], non-contact air-coupled excitation [11] and by lateral pushing the particles in a SEM-like set-up [10]. The existence of elastic rolling resistance is now well established. A non-contact/non-invasive method for determining the CLA prior to freerolling has been reported recently [12].

In current study, we detail an experimental approach to acquire CLA data for a set of PSL microparticles in a non-contact manner. In the reported experiments, Rayleigh surface acoustic waves (SAW) are utilized as a precise excitation mechanism and, image processing and interferometry are used as monitoring and detection techniques for capturing the micro-/nano-scale motions and vibrational responses of the microparticles base-excited by a prescribed SAW field. In order to determine the CLA in a systematic manner, the amplitude, frequency and duty cycle of the applied SAW field are designed as a precisely controlled excitation mechanism to transfer rotational momentum to the microparticles deposited on a dielectric substrate in a controllable manner. The adhesion properties of the particles were extracted from their transient rocking responses, acquired by a laser Doppler vibrometer (LDV), which also was used for characterizing the SAW field by measuring the out-of-plane displacement of the substrate surface. In addition, the micro-scale 
drifting motion (rolling and/or sliding) of the particles was captured by a CCD camera integrated with the monitoring microscope and was image-processed for determining their trajectories. The term drift motion is used when the precise nature of the particle motion is unknown. Drift motion can be particle rolling, sliding or flying, or a combination of all, but not rocking. It is observed that, as expected, in general, particles roll/move towards the source of the SAW field.

\section{Background}

When subjected to an external force/moment effect, the adhesion bond of a spherical particle on a flat elastic substrate creates both a restitution force (out-of-plane) and a moment resisting the outof-plane displacement and leaning/rocking (in-plane) angle of the particle, respectively [9]. Based on the Johnson-Kendall-Roberts (JKR) adhesion model [6] and as previously reported [12,13], the natural frequencies for the out-of-plane and rocking motions ( $f_{\mathrm{o}}$ and $f_{r}$, respectively) of a spherical particle with a radius of $R$, mass density of $\rho$ and the particle-substrate interface work-of-adhesion of $W_{A}$ are related by:

$$
f_{o}=\frac{1}{2 \pi} \sqrt{\frac{27}{20} \frac{1}{\rho R^{3}}\left(\frac{3 W_{A} K^{2} R^{2}}{4 \pi^{2}}\right)^{1 / 3}} \text { and } f_{r}=\frac{1}{2 \pi} \sqrt{\frac{45}{14} \frac{W_{A}}{\rho R^{3}}}
$$

where $K=\frac{4}{3}\left(\left(1-v_{p}^{2}\right) / \mathrm{E}_{p}+\left(1-v_{s}^{2}\right) / \mathrm{E}_{s}\right)^{-1}$ is the stiffness coefficient of the particle-substrate adhesion bond, $E_{s}$ and $E_{p}$ the Young's moduli and $v_{s}$ and $v_{p}$ the Poisson's ratios of the substrate and the particle materials, respectively. When a particle is subjected to an external lateral force or excitation field, the leaning angle of the particle increases by increasing the external excitation force/moment [10]. When the leaning angle of the particle reaches a critical value that the resisting moment of the 
adhesion bond is unable to restore the particle to its original stable equilibrium position, the substrate-particle adhesion bond breaks and the particle detaches from the substrate and begins to drift on the substrate. Depending upon the adhesion force, friction and/or magnitude of the moment, the particle rolls, slides, or glides/flies away from its contact point.

The critical leaning angle is crucial in many processes as it marks the time instant when the particle motion is initiated (often in an unstable manner). For determining the CLA of a particle, the external forces and the resulting resisting rolling moment acting on the particle at the onset of initiation of the particle motion are required. The restitution moment is generated by the lateral force acting on the particle arising from the in-plane displacement of the substrate due to the induced SAW field $(\Phi(x, t))$ (Fig. 1.a). Since the amplitude of the lateral displacement $\left(u_{x}\right)$ and the out-of-plane displacements $\left(u_{y}\right)$ of the substrate under a SAW field influence are close to each other [14], their second derivatives are expected to be close too, hence the magnitudes of the lateral force $\left(F_{x}\right)$ and the out-of-plane force $\left(F_{y}\right)$ acting on the particle can be assumed to be approximately equal. To calculate the out-of-plane force acting on the particle resulting from the contact base excitation, the out-of-plane acceleration of the particle needs to be calculated. For a particle undergoing a harmonic motion under the influence of the SAW field, the motion equation can be simply written as:

$$
y=A \cos (\omega t-\varphi)=\frac{u_{y p p}}{2} \cos \left(2 \pi f_{c} t-\varphi\right)
$$

where $A$ is the oscillation amplitude and can be substituted by $A=u_{\mathrm{ypp}} / 2$ where $u_{\mathrm{ypp}}$ is the peak-topeak amplitude of the oscillation, $\omega=2 \pi f_{\mathrm{c}}$ is the angular frequency of the motion and $f_{\mathrm{c}}$ is the base- 
excitation frequency. The acceleration of the motion is obtained by taking the second derivative of the acceleration with respect to time as:

$$
a=\frac{d^{2} y}{d t^{2}}=-2 u_{y p p}\left(\pi f_{c}\right)^{2} \cos (2 \pi f t-\varphi)
$$

From Eq. (3) the maximum amplitude of the out of plane acceleration of the harmonic motion of the substrate under the influence of the SAW field is approximated as:

$$
a_{y}=|a|=2 u_{y p p}\left(\pi f_{c}\right)^{2}
$$

The maximum out-of-plane force acting on the particle is $F_{y}=m_{p} a_{y}$. By substituting the particle mass as $m_{p}=4 / 3 \pi \rho R^{3}$ and the acceleration from Eq. (4), the maximum out-of-plane harmonic force acting on the particle is approximated as:

$$
F_{y} \cong \frac{16 \pi^{3}}{3} u_{y p p} \rho f_{c}^{2} R^{3}
$$

The maximum rolling moment resulting from a lateral force $\left(F_{x}\right)$ with respect to the particlesubstrate contact point (point $C$ in Fig. 1.a) then becomes:

$$
M_{c z}=F_{x} R \cong F_{y} R \cong \frac{16 \pi^{3}}{3} u_{y p p} \rho f_{c}^{2} R^{4}
$$


Assuming a linear bending stiffness of the adhesion bond, the leaning angle $\left(\theta_{l}\right)$ and the rolling moment of the particle are related by the following relationship:

$$
M_{c z}=k_{b} \theta_{l}
$$

where $k_{b}$ is the equivalent bending stiffness of the bond. In Ref. 8, based on Ref. 7, the bending stiffness of the bond is provided as a function of the radius $(R)$ the rocking frequency $\left(f_{r}\right)$, and the mass density $(\rho)$ of the particle as:

$$
k_{b}=\frac{64}{5} \pi^{3} \rho f_{r}^{2} R^{5}
$$

By substituting the relationship for rocking frequency from Eq. (1) into Eq. (8), the bending stiffness of the bond is expressed as a function of the work-of-adhesion of the particle-substrate pair:

$$
k_{b}=\frac{72}{7} \pi W_{A} R^{2}
$$

As discussed above, the rolling moment and leaning angle of the particle reach their critical levels at the onset of the breakage of the particle-substrate bond and the initiation of the particle drifting. The corresponding rolling moment and leaning angle are called the critical rolling moment $\left(M_{c z}^{c r}\right)$ and critical leaning angle $\left(\theta_{l}^{\text {cr }}\right)$, respectively. Here we assume that the drifting of the particle on the substrate occurs as a no-slip motion and initially the particle makes no sliding motion on the 
substrate. Using Eqs. (6), (7) and (9), the CLA of the particle at the onset of its free-drifting is expressed in terms of the experimentally determined quantities $\left(u_{y p p}, f_{c}\right.$ and $\left.W_{A}\right)$ and known particle property parameters $(R$ and $\rho)$ as:

$$
\theta_{l}^{c r}=\frac{14}{27} \pi^{2} \frac{u_{y p p} \rho f_{c}^{2} R^{2}}{W_{A}}
$$

To obtain the critical angle, in the experimental procedure detailed below, the displacement amplitude $u_{y p p}$ is linearly increased by increasing the amplitude of the SAW field until particle detachment and particle motion is observed. The corresponding angle is calculated using Eq. (10) and reported as the critical leaning angle $\theta_{l}^{c r}$ for a specific particle at a particular contact point.

\section{Experimental Setup and Procedure}

The main objective of the current experimental work is to accurately determine the leaning angle of a single microparticle prior to the initiation of its free-rolling motion under the influence of contact base-excitation generated by a transient displacement field of a prescribed SAW pulse. A two-step non-contact/non-invasive experimental approach is developed and employed for observing the particle vibrational dynamics and motion simultaneously under prescribed Rayleigh SAW field and determining the CLA of a microparticle at the onset of rolling. After cleaning the substrate, PSL microparticles are dry-deposited on a soda lime substrate and then the CLA determination experiment is carried out via SAW induced drifting. An SEM (Scanning Electron Microscopy) image of the particle is included, indicating substantial surface roughness (Fig. 1.d). Finally, the insitu determination of the average work-of-adhesion of the particles is conducted via the non- 
invasive ultrasonic excitation and laser interferometry sensing techniques developed previously $[5,9,12,13,15]$ (Fig. 1.b). The adhesion properties of the microparticles are extracted from their transient rocking motion response (Eq. 1). The set-up is also utilized for characterizing the SAW field by measuring the out-of-plane displacement of the substrate surface. The videos of drifting particles were captured by a digital camera integrated with a monitoring optical microscope. The obtained video files are image-processed for determining the particle trajectories and their characteristics.

\subsection{Materials and Sample Preparation}

The microparticles utilized in the reported experiments are NIST (National Institute of Standards and Testing) traceable polystyrene latex (PSL) spherical microparticles (DC-15 DryCal, Thermoscientific, Waltham, Massachusetts, USA) with a diameter of $14.9 \pm 0.6 \mu \mathrm{m}$, a mass density of $\rho=1050 \mathrm{~kg} / \mathrm{m}^{3}$ and an average particle mass of $m=1.86 \mathrm{ng}$. The particles are dry-deposited on a soda-lime glass photomask substrate $(6 \times 6 \times 0.25 ")$ using a pipette tip. Prior to deposition of the particles, the soda-lime glass substrate is cleaned in a four-step process. Initially, the substrate is washed and rinsed with DI (de-ionized) water. Then, acetone is used for rinsing the substrate to remove organic residuals from its surface, and the substrate is finally washed and rinsed with a $2 \%$ Hellmanex II solution. Following the final rinsing, the substrate is dried by compressed dry air.

\subsection{Waveform Design and SAW Excitation}

To be able to precisely control the amount of energy, force and moment supplied to the particles, the excitation pulses are designed in a way to controllably produce the desired surface acoustic wave field on the substrate. The instruments used in the current experimental set-up (Fig. 1.b) for 
producing the excitation pulses for creating the SAW field on the substrate include a function generator (DS345, Stanford Research Systems, Sunnyvale, California, USA) employed as the trigger source, a Lin/Log sweep generator (185, Wavetek Inc., San Diego, California, USA) that produces the excitation pulses and a RF amplifier (A300 RF, Electronics \& Innovation, Ltd. Rochester, New York, USA) that amplifies the excitation pulses to a desired level with amplification factor of $A_{f}=55 \mathrm{~dB}$ (Fig. 2). The generated high voltage pulses are consequently fed to a commercial ultrasonic transducer (AT024, CTS Corporation, Albuquerque, New Mexico, USA) with a central frequency of $f_{c}=2.25 \mathrm{MHz}$ integrated with an acoustic wedge $\left(\theta_{w}=42.2^{\circ}\right)$ made of Rexolite ${ }^{\circledR}$ with the mass density of $1049 \mathrm{~kg} / \mathrm{m}^{3}$ mounted on the soda-lime glass substrate using double-sided tape to create SAW field on the substrate surface. The SAW inducting angle of the wedge $\left(\theta_{w}\right)$ is designed to be $\theta_{w}=90^{\circ}-\sin ^{-1}\left(c_{L} / c_{R}\right)=42.2^{\circ}$, based on Snell's law, with the longitudinal (pressure) wave speed of $c_{L}=2337 \mathrm{~m} / \mathrm{s}$ in the Rexolite ${ }^{\circledR}$ wedge material and the Rayleigh wave speed of $c_{R}=3188.4 \mathrm{~m} / \mathrm{s}$ in the soda-lime glass substrate.

In the reported experiments, the designed excitation pulses for producing SAW field on the substrate surface were applied to be able to determine the CLA of the particles in a systematic manner. The excitation of the ultrasonic pressure transducer for creating the SAW field on the substrate takes place in 21 sequential burst cycles $\left(N_{b}^{\max }=21\right)$. Each triggering burst cycle includes a train of triggering pulses with a duty cycle of $D=75 \%$, an active duration of $t_{a}=15 \mathrm{~s}$, a passive duration of $t_{p}=5 \mathrm{~s}$, and a burst period of $T_{b}=t_{a}+t_{p}=20 \mathrm{~s}$. A triggering pulse is generated during the active time of the burst cycle. At the passive time of the burst cycles, no triggering pulse is generated, allowing the particles sufficient time to re-settle prior to the start of the next burst cycle. The trigger source for the prescribed excitation is the function generator that delivers square pulses 
with an amplitude of $V_{t p}=+4.5 \mathrm{~V}$ with a frequency of $f_{t p}=500 \mathrm{~Hz}$ (period of $\left.T_{t p}=2 \mathrm{~ms}\right)$. The number of triggering pulses-per-burst cycle is therefore $N_{p b}=t_{a} \times f_{t p}=7500$. The sweep generator is triggered with the triggering pulses, and at each triggering pulse (event) it generates a square excitation pulse with a pulse frequency of $f_{p}=2.25 \mathrm{MHz}$ (equal to the central frequency of the ultrasonic transducer $\left(f_{c}\right)$ ) and an amplitude voltage of $V_{p}$ which is increased manually by a voltage increment of $\Delta V_{p} \cong 30 \mathrm{~V}$ at the start of each successive burst cycle, during the passive time $\left(t_{p}\right)$, up to $V_{e p p}^{\max }=643.4 \mathrm{~V}$ occurring at $N_{b}^{\max }=21$. After being increased at the passive time, the amplitude of the excitation pulses $\left(V_{p}\right)$ is kept constant throughout the active time $\left(t_{a}\right)$ of the burst cycles.

\subsection{Data Acquisition}

The current experimental setup acquires the micrometer-scale drifting motion of the particles as well as their nanometer-scale vibrational dynamics, simultaneously, under the influence of a predefined Rayleigh SAW field. The micrometer-scale drifting motion of the particles is observed and captured using a CCD (charge-coupled device) camera (A102f, Basler, Ahrensburg, Germany) with a frame rate of $30 \mathrm{fps}$ integrated into a monitoring optical microscope (BXFM-ILHSPU, Olympus, Tokyo, Japan) with a $5 \times$ optical objective in a field-of-view of $2380 \times 1520 \mu \mathrm{m}$ (Fig. 1). Throughout the experiment, the drifting motion of the microparticles within a fixed field-of-view is acquired and saved in video files for tracking the lateral drifting of the particles subjected to the controlled SAW field (Fig. 3.a). Image processing is performed on the recorded video files using the Mosaic/Particle Tracker plugin of the ImageJ software (V1.48, National Institution of Health, Bethesda, Maryland, USA) to analyze the micro-scale motion of the particles in order to detect the initiation of the substrate-particle bond breakage (for determining CLA) and to determine the trajectories of the drifting of the particles under the influence of the prescribed SAW field. 
The nanometer-scale vibrational dynamics (i.e., the out-of-plane responses of the particle apexes and the substrate surface) are captured using a laser Doppler vibrometer (LDV) setup. The LDV setup consists of a laser fiber interferometer (OFV511, LDV, Polytec, Waldbronn, Germany) and a vibrometer controller (OFV3001, Polytec, Waldbronn, Germany) that are integrated with the optical microscope to detect the transient out-of-plane displacements of the substrate and the microparticles at a sub-nanometer theoretical resolution (less than $1 \AA$ ) in the displacement amplitude range of $\pm 75 \mathrm{~nm}$ and frequency range of $50 \mathrm{kHz}$ to $35 \mathrm{MHz}$ (Fig. 1.a). The detected transient out-of-plane displacement responses of the particles and the substrate in their temporal domain are acquired, digitized and averaged employing a digitizing oscilloscope (TDS 3052, Tektronix, Beaverton, Oregon, USA) with a frequency bandwidth of $500 \mathrm{MHz}$ (Fig. 1.b). The acquired time series (waveforms) are subsequently saved in a personal computer for further spectral analysis to accurately measure and characterize the propagating SAW field on the substrate and to determine the adhesion characteristics of the particles by adopting previously reported methods [8-12].

The out-of-plane response of the substrate to the propagating SAW field at each burst cycle (Fig. 2.a) is acquired with the laser probe of the LDV focused on the substrate at the center of the monitoring area. The out-of-plane responses of the substrate $\left(u_{y}(\mathrm{t})\right)$ for five different burst cycles are shown in Fig. 2.b. The overall shape of the substrate response waveform stays nearly identical during each burst cycle and only the amplitude of the response is increased with the increasing excitation pulse voltage as expected, indicating linearity of the responses. The variation of peak-topeak amplitudes $\left(u_{y p p}\right)$ of the out-of-plane response of the substrate during the experiment at different burst cycles is shown in Fig. 2.c. 
After completing the burst cycles and acquiring the drifting motion responses of two sample sets with 30 particles in each (Sample Sets A and B) and measuring the transient out-of-plane response of the substrate during each cycle, the out-of-plane responses of a set of ten-particles in Sample Set A within the monitoring area of the substrate are acquired for determining the average work-ofadhesion between the PSL particles and the soda-lime glass substrate (Fig. 2.d). This work-ofadhesion is required for calculating the CLA of the particles in Sample Set A (Eq. (10)). Sample Set B is used only for demonstrating repeatability and particle motion stability of the experimental setup and the reported approach substrate (Fig. 3.b). The transient out-of-plane displacement responses of the particles in Sample Set A and the substrate surface at their neighboring points are acquired, digitized, and averaged employing the LDV setup and the digitizing oscilloscope. The acquired raw temporal domain responses (waveforms) of the particles and the substrate are processed into their spectral domain response, using a Fast Fourier Transform (FFT) routine, and then compared together for determining the rocking resonance frequencies of the particles (Figs. 2.d).

\section{Results and Discussions}

The micrometer-scale dynamics of a sample set of 30 PSL particles in Sample Set A, dry deposited on a soda lime glass substrate was acquired during 21 successive burst cycles of prescribed SAW field to determine the CLA of single microparticles prior to the initiation of their drift motion on the substrate. Each particle in the sample set is tracked throughout the experiment while the amplitude of the excitation was increased gradually at the start of each burst cycle. The average work-ofadhesion values for a sample set of ten particles were determined by acquiring and analyzing their nanometer-scale rocking motion on the substrate and were used in CLA determination calculations. 
the trajectories of all of the particles in Sample Set A throughout the experiment during 21 successive burst cycles are acquired and depicted (Fig. 3.a). For establishing the stability of the setup, the particle trajectories for Sample Set B are also obtained with a different transducer arrangement (Fig. 3.b). Predominantly, the trajectories of the particles are in the same general direction; towards the SAW source and nearly at the angle of the incoming SAW fronts. The motion trajectories of the particles (Sample Set A) in Figs. 3.a-c are determined by imageprocessing the experimentally acquired video files of their dynamics. The total travel distances made by the particles during each excitation burst cycle vary significantly from each other, ranging from a few micrometers to approximately $2 \mathrm{~mm}$. Close ups of the trajectories of three particles of Sample Set A $\left(N_{p}=26,29\right.$ and 30) and two particles of Sample Set B $\left(N_{p}=17,30\right)$ are shown in Figs. 3.c-f, with the circled numbers indicating the locations of the particles at the beginning of particular SAW burst cycles $\left(N_{b}\right)$.

When a particle starts to move for the first time or after it has been stationary (immobile) on the surface for the duration of one or more burst cycles, the initiation of motion is marked as a translational (drifting) motion onset incident (MOI) (for example see Fig. 3.c for particles 29 and 30 of Sample Set A). When a particle starts its motion on the substrate for the first time (MOI 1), it indicates that because of increased excitation amplitude, the leaning angle of this previously rocking particle has reached and passed its critical value. When the CLA of the particle is reached, it departs its elastically stable state of rocking motion and starts to drift by breaking (rupturing) its bond with the substrate. When the excitation period of the burst cycle expires, the particle stops its motion during the passive time. If the particle stays immobile for at least one full active time of the next burst cycle, by the time it resumes its drifting again at a later burst cycle, the next MOI event 
occurs. A new MOI indicates yet another occurring of CLA for the particle, different from the first observed one at MOI 1.

As shown in Figs. 3.b-c, almost all of the particles in Sample Set A alter their drifting trajectories at the start of a new MOI. This alteration in trajectory direction of a particle could indicate that when the particle becomes immobile for at least one complete burst cycle (at least 20 seconds), the adhesion characteristics at its contact zone change, leading to the change in the direction of the particle motion when it starts to drift again at later burst cycles. Investigating the trajectories of the particles reveals that they exhibit different responses to the same excitation field. At the same excitation level some of the particles start/continue drifting while others remain/become immobile. For example, at the seventh burst cycle $\left(N_{b}=7\right)$, almost $10 \%$ of the particles experience MOI $1,3 \%$ experience MOI 2, $10 \%$ stop drifting and go into stationary state, $30 \%$ continue to drift and the remaining of the particles $(47 \%)$ remain immobile. This example illustrates that the burst cycles that MOI 1 and/or subsequent additional MOI events occur, vary for each particle. The difference in the patterns of the micrometer-scale dynamic behavior of the particles at the same excitation condition indicates that the drifting motion of a particle is not only a function of the excitation SAW field and its characteristics, but also a function of the local surface properties of the particle and the substrate at the contact zone, the trajectory/history of the motion and possibly the surface electrostatic charges. Similar trajectories are obtained for the particles in Sample Set B (Figs. 3.d-e).

A bar graph of the stop/drift pattern of the particles in Sample Set A is presented in Fig. 4.a. Particles frequently start/stop their drifting at various burst cycles in response to a controlled SAW excitation. For example, particle $20\left(N_{p}=20\right)$ starts its motion for the first time at burst cycle $N_{b}=5$ (MOI 1), then stops at $N_{b}=6$, restarts drifting at $N_{b}=7$ (MOI 2) and continues to drift until $N_{b}=10$ when it goes into stationary state for 5 sequential burst cycles. The particle later restarts its motion 
again at $N_{b}=15$ (MOI 3), drifts just for one cycle, stops again at $N_{b}=16$ and remains immobile for three burst cycles until it resumes its motion at $N_{b}=19$ (MOI 4) and continues to drift for the next three burst cycles until the end of the experiment. In Fig. 4.a, the solid bars represent the time that a particle is immobile while the hatched bars indicate the time that the particle drifts on the substrate. In this figure, the end of a stationary bar and start of a motion bar shows the occurrence of a MOI for the corresponding particle. Some of the motion/stop bars end before all the 21 burst cycles complete (particles $N_{p}=2,3,4,6,9,11,14,17,18,19,23$, and 24) that are related to the particles that roll out of the monitoring area before the end of all 21 burst cycles.

It is observed that each particle illustrates unique pattern of drifting motion during the experiment, largely attributed to the surface morphologies of particles (Fig. 1.d) and the substrate. Less than $20 \%$ of the particles in Sample Set A stop at the middle of the active time of a burst cycle, that could be attributed to surface imperfection of the particles and the substrate and/or developed electrostatic charges on the particles due to triboelectric effects. It is already reported that the electrostatic charges have a considerable effect on the adhesion of microparticles [16]. The inhomogeneity in the surface properties of the soda lime glass substrate (see [17]) is believed to be another major parameters causing the scattering in the results of CLA for different particles in Sample Set A. All of the particles almost instantly halt their motion when the passive time $\left(t_{\mathrm{p}}\right)$ of a burst cycle starts. This behavior indicates that the adhesion forces between the particles and the substrate (van der Waals, electrostatic, etc.) are strong enough to immediately overcome the inertia of a previously drifting particle, in the absence of SAW field. When sufficiently long resting time $\left(t_{p}=5 \mathrm{~s}\right)$ is allowed for the particles, it is observed that a number of them settle down in their new locations on the substrate to develop new strong bonds with the substrate. When subsequently the active time of the burst cycle begins with higher excitation amplitude, some of the particles resume 
their motion while others remain immobile. For example, particle $N_{p}=5$ of Sample Set A starts to drift at $N_{b}=7$, then stops at the start of $N_{b}=8$ for the duration of the passive time ( $5 \mathrm{sec}$ ), but it is unable to drift again at $N_{b}=8$ even though the excitation amplitude is higher than previous burst cycle (Fig. 4.a). On the other hand, while both particles $N_{p}=1$ and 5 similarly start to drift at $N_{b}=7$ and stop during the passive time of burst cycle $N_{b}=8$, yet when the active time begins, particle $N_{p}=1$ starts to drift again while particle $N_{p}=5$ stays immobile. Particle $N_{p}=1$ continues to drift during the next 5 burst cycles until at the start of $N_{b}=13$ it becomes immobile too (despite experiencing the highest excitation amplitude, so far, at this cycle). Particle $N_{p}=1$ stays immobile during next 4 burst cycles till it starts to drift again at $N_{b}=17$. This observation indicates that for some of the particles, $t_{p}=5 \mathrm{~s}$ is a sufficiently long time period to form strong bonds with the substrate to immobilize a previously drifting particle even under higher excitation amplitude.

The percentage of the drifting particles during different burst cycles is presented in Fig. 4.b. This figure indicates that the particles do not start to drift on the substrate until the excitation amplitude passes the $V_{e p p}=100 \mathrm{~V}$ threshold. Then, as the excitation pulse voltage increases cycle by cycle, the number (percentage) of the drifting particles increases from burst cycles $N_{b}=3$ to 5 (as $V_{e p p}=100 \rightarrow$ $150 \mathrm{~V})$. This is expected because, by increasing the excitation amplitude, the amount of the energy delivered to the particles increases, enabling more of them to break their bonds with the substrate and to start drifting. Afterwards the percentage of the drifting particles start to decrease or stay almost constant (with some fluctuations) as the number of the burst cycles and the excitation amplitude increases, which is counterintuitive at the first glance, since it was still expected that by further increasing the excitation amplitude and providing higher energy for the particles, more of them would be able to break their bonds and start drifting. The decline in the percentage of drifting particles while the excitation amplitude increases, indicates that the particle-substrate bonds become 
stronger by increasing the excitation amplitude and prevent the particles from breaking their bond with the substrate even with higher available dynamic energy. The increase in the strength of the particle-substrate bonds can be attributed to the triboelectric effect. It is hypothesized that the particles that started to drift on the substrate at earlier burst cycles begin to develop electrical charges on their surface, in particular, and on the surface of the substrate, in general, due to triboelectric effect, arising from the drifting on the substrate. The developed electrical charges on both surfaces could increase the adhesion forces between the particles and the substrate, making it more difficult for the particles to break these stronger bonds even with higher dynamic energy supplied to them by increasing the excitation amplitude. As the excitation amplitude further increases, some of the previously drifting particles stop because of developed electric charges on their surfaces while some new particles, with no electrical charges on them yet, start to drift for the first time and then they too develop electrical charges on their surfaces and subsequently stop moving after a few burst cycles. In total the number of the particles drifting on the substrate stays almost constant with some fluctuations as the excitation amplitude increases from $V_{e p p}=150 \mathrm{~V}$ to $500 \mathrm{~V}$ (at burst cycles $N_{b}=6$ to 16 ). Eventually, by further increasing the excitation amplitude, the dynamic energy provided for the particles becomes vigorous enough to overcome and dominate the reverse effect of the developed triboelectric electrical charge and to increase the number of the particles that are able to break their bonds. After the excitation amplitude threshold of $V_{e p p}=500 \mathrm{~V}$ is reached (at $N_{b}=16$ ), the percentage of the drifting particles increases as the excitation amplitude continues to surge indicating that the increased supplied dynamic energy via the SAW field has dominated the triboelectric effect. It seems that a third degree polynomial fit (dotted line in Fig. 4.b) is able to adequately follow the trend of the percentage of the drifting particles versus excitation amplitude. Investigating the equation of the fitted cubic polynomial (Fig. 4.b) indicates that the 
second degree indeterminate $\left(V_{e p p}^{2}\right)$ has a negative coefficient, possibly related to the triboelectric effect. From this observation one might hypothesize that in this particular case the effect of triboelectric charge on the percentage of drifting particles is negatively proportional to the square of the excitation amplitude. Further experimental and analytical investigations is needed to accurately formulate, characterize and explain the developed triboelectric charge on the particles.

The CLA $\left(\theta_{l}^{c r}\right)$ of the particles in Sample Set A at each MOI is calculated by substituting the experimentally acquired excitation characteristics $\left(u_{y p p}, f_{c}\right)$ and the adhesion properties $\left(W_{A}\right)$ alongside with the known properties of the particles $(\rho, R)$ into the developed mathematical model (Eqs. 1-10). The CLA results for the studied particles are demonstrated in Fig. 4.c. Note that CLA for the particles in the sample set, range from $0.9^{\circ}$ to $7.8^{\circ}$. From Fig. 4.c, it is observed that the particles could be categorized in three distinctive groups based on their CLA at the first MOI: Group I for particles with CLA between 0.9 and $1.2^{\circ}$ (47\% of the sample set), Group II between 2.0 and $4.2(30 \%)$ and Group III between 5.3 to $7.8^{\circ}(20 \%)$. In Figs 4.a and 4.c the particles are shown categorized and sorted by their group numbers (Groups I, II and III). The distribution in Fig 4.c demonstrates that the particles from the same group do not necessarily exhibit consistent dynamic behavior (starting to move, drift or stop drifting) after MOI 1, except that the CLAs in MOI 2 for Group I are higher than those for Group II. It is concluded that the dynamic behavior of a particle is not merely a function of its surface properties, but also a function of the local surface properties of the substrate.

To better understand the effect of the local substrate surface characteristics on the drifting of the particles, the group number of the particles in Sample Set A is shown by superscript Greek numbers next to the particle number in Fig. 3.a. This figure clearly shows that some of the particles that are 
located close to each other on the substrate are categorized in different groups. For instance, the neighboring particles 14,15 and 16 , located close to each other at the start of the experiment, start their drifting motion at burst cycles dissimilar to each other and their CLAs in MOI 1 are so diverse that makes them categorized into three different groups. Note that as indicated by Fig 3.a, particles of each group are distributed uniformly on the substrate and are not aggregated in a particular section of the substrate. It seems that the location of a particle on the substrate exhibits virtually no correlation with its CLA and the group it belongs to. This indicate that the regular nodal patterns from wave interference are not regarded to be the main mechanism for particle motion halting. It is hypothesized that the CLA of a microparticle is a function of the surface properties of both the particle and the substrate at the contact point and not merely the excitation mechanism.

In Fig. 5, a histogram representation of the distribution of the CLAs of the Sample Set A particles in all MOIs is illustrated. It demonstrates the CLA occurrence frequency $\left(f_{\theta_{l}^{c r}}\right)$ of the particles over the range of $\quad \theta_{l}^{c r}=0-9.0^{\circ}$ divided into bins with a width of $\Delta \theta_{l}^{c r}=0.3^{\circ}$. It is clearly observable that the majority of CLA occurrences fall between $0.9^{\circ}<\theta_{l}^{\text {cr }}<1.2^{\circ}$ in MOI $1(47 \%)$. The $\theta_{l}^{\text {cr }}$ occurrences for MOI 2-5 are distributed over the range of $\theta_{l}^{c r}=2.0-9.0$ degrees. Considering only the CLAs at MOI 1, the histogram presents a unimodal skewed right distribution pattern with the dominant mode at the bin of $0.9-1.2^{\circ}$. This pattern indicates that the majority of the particles start their motion for the first time when their leaning angle reaches the range of $\theta_{l}^{c r}=0.9-1.2^{\circ}$ suggesting this range to be the main observed CLA range for the studied particles.

A summary of the acquired results for the thirty-particle sample set are tabulated in Tables 1 and 2 . Table 1 lists the burst cycle numbers $\left(N_{b}\right)$, associated peak-to-peak substrate response amplitudes 
$\left(u_{y p p}\right)$ and CLAs $\left(\theta_{l}^{c r}\right)$ of the Sample Set A particles at different MOIs, while Table 2 categorizes these data into three groups (I, II and III) for the first MOI. The main measure of CLA is extracted from MOI 1, where subsequent MOI events are viewed as capture release events extraneous to the MOI 1. The MOIs following the MOI 1 are due to capture release of defective substrate/surface interfaces at higher stresses, rather than a measurement of ideal particle-substrate interaction. Note that the MOI 2 through MOI 5 events are decreasing in population, with MOI 5 representing just a single particle supporting the implication that the principal CLA occurs at MOI 1. The critical leaning angle values (especially at MOI 1) are taken to be a quantitative metric of particle-substrate interaction by indicating the quality of the bonding present with a measurement of a bond rupture incident.

\section{Conclusions and Remarks}

In current work, a non-contact acoustic technique is introduced for acquiring the critical leaning angle (CLA) of individual microparticles under precisely-controlled base-excitation conditions. Two sample sets with 30 particles each are utilized. For the first sample set of 30 particles (Sample Set A), it is found that the CLAs of $47 \%$ of the microparticles in the sample set fall between $0.9^{\circ}$ to $1.2^{\circ}$ (Group I) while $30 \%$ of the particles have the CLAs between $2.0^{\circ}$ and $4.2^{\circ}$ (Group III) and $20 \%$ between $5.3^{\circ}$ to $7.8^{\circ}$ (Group III). Only $3 \%$ of the particles in the sample set did not exhibit any drifting motion. Sample Set B is used to demonstrate the repeatability and stability of the set-up and the presented approach. An analysis of the trajectories of the particles motion in the two sample sets indicates that in general a majority of the microparticles tend to move towards the SAW field source. They, however, could change their direction, speed and acceleration and stop/restart their drifting motion at different excitation amplitudes, indicating a possible inhomogeneity of the 
surface properties of the particles and the substrate, and electrical charge distributions on their surfaces. The electric charge distribution on a particle could be due to its initial electrical charge and/or a developed electrical charge during the experiment due to the triboelectric effect. In any case, the behavior of a bond rupture event in the form of a MOI is utilized as an event for determining a metric of particle-surface bond characterization, namely a CLA, quantitatively measuring the bond quality between particle-surface in a non-contact manner. Understanding the microparticle rolling resistance is essential in several industrial and biological processes such as blood flow, cell sorting, drug delivery, rolling of biological cells, pharmaceutical production, chemical mechanical polishing in semiconductor industry, and the adhesion of toner/ink particles in printing/copying. In addition, CLA is a critically important parameter for improving the accuracy of computational simulations of granular systems and particles networks. 


\section{ACKNOWLEDGMENTS}

The authors gratefully acknowledge financial support through grants from the National Science Foundation (NSF) (Award Numbers: 1066877 and 1200839). Partial funding was provided by Clarkson University and CAMP (Center for Advanced Materials Processing). Thanks are due to Dr.

Grazyna Kmiecik-Lawrynowicz, Dr. Santokh Badesh, of Xerox Corp. and Dr. Maura Sweeney of Stratasys, Inc. for stimulating discussions and guidance. 


\section{REFERENCES}

[1] L.M. Cook, Chemical processes in glass polishing, J. Non-Cryst. Solids. 120 (1990) 152-171.

[2] H. Mizes, M. Ott, E. Eklund, D. Hays, Small particle adhesion: measurement and control, Colloids Surf. Physicochem. Eng. Asp. 165 (2000) 11-23.

[3] A.L. Dunehoo, M. Anderson, S. Majumdar, N. Kobayashi, C. Berkland, T.J. Siahaan, Cell adhesion molecules for targeted drug delivery, J. Pharm. Sci. 95 (2006) 1856-1872.

[4] M.A. Bewick, R.M. Lafrenie, Adhesion dependent signalling in the tumour microenvironment: the future of drug targetting, Curr. Pharm. Des. 12 (2006) 2833-2848.

[5] M.M. Peri, C. Cetinkaya, Non-contact microsphere-surface adhesion measurement via acoustic base excitations, J. Colloid Interface Sci. 288 (2005) 432-443.

[6] K.L. Johnson, K. Kendall, A.D. Roberts, Surface energy and the contact of elastic solids, in: Proc. R. Soc. Lond. Math. Phys. Eng. Sci., The Royal Society, 1971: pp. 301-313.

[7] K.L. Johnson, J.A. Greenwood, An adhesion map for the contact of elastic spheres, J. Colloid Interface Sci. 192 (1997) 326-333.

[8] C. Dominik, A. Tielens, Resistance to rolling in the adhesive contact of two elastic spheres, Philos. Mag. A. 72 (1995) 783-803.

[9] M.M. Peri, C. Cetinkaya, Rolling resistance moment of microspheres on surfaces, Philos. Mag. 85 (2005) 1347-1357.

[10] W. Ding, A.J. Howard, M.M. Peri, C. Cetinkaya, Rolling resistance moment of microspheres on surfaces: contact measurements, Philos. Mag. 87 (2007) 5685-5696.

[11] M.M. Peri, C. Cetinkaya, Air-coupled excitation of rocking motion of individual microspheres on surfaces, Appl. Phys. Lett. 90 (2007) 171906. 
[12] B. Farzi, C.K.P. Vallabh, J.D. Stephens, C. Cetinkaya, Critical rolling angle of microparticles, Appl. Phys. Lett. 108 (2016) 111602. doi:10.1063/1.4944043.

[13] I. Akseli, M. Miraskari, H. Zhang, W. Ding, C. Cetinkaya, Non-contact rolling bond stiffness characterization of polyvinylpyrrolidone (PVP) particles, J. Adhes. Sci. Technol. 25 (2011) $407-434$.

[14] K.L. Mittal, R. Jaiswal, Particle adhesion and removal, John Wiley \& Sons, 2015.

[15] C.K.P. Vallabh, J.D. Stephens, C. Cetinkaya, Effect of surface temperature on microparticlesurface adhesion, Appl. Phys. Lett. 107 (2015) 41607.

[16] C.K.P. Vallabh, A.S. Vahdat, C. Cetinkaya, Charge contribution to patch-charged microparticle adhesion, Appl. Phys. Lett. 105 (2014) 211905.

[17] S. Rizvi, Handbook of photomask manufacturing technology, CRC Press, 2005. 


\section{FIGURE CAPTIONS}

Figure 1. (a) The leaning of a microparticle under the influence of a SAW field. (b) Instrumentation and connectivity diagram of the experimental set-up. Not to scale. (c) Close-up of the monitoring zone of the experimental set-up. (d) SEM image of microparticles utilized in the reported experiments, depicting surface morphology.

Figure 2. (a) A train of triggering burst cycles generated by the function generator. Each burst cycle consists of a train of triggering square pulses with a burst period of $T_{b}=20 \mathrm{sec}$ with a passive time duration of $t_{\mathrm{p}}=5 \mathrm{sec}$, active time duration of $t_{\mathrm{a}}=15 \mathrm{sec}$ and duty cycle of $\mathrm{D}=75 \%$. (b) Transient responses of the substrate $\left(u_{y}(t)\right)$ to excitation pulses for five specific burst cycles $\left(N_{b}=1\right.$, $4,8,16$ and 21) with the corresponding peak-to-peak excitation pulse voltages of $V_{e p p}=35.6,73.1$, 176.2, 405.2 and 643.4 V, respectively. Inset to Fig. 2.b: The spectral domain response of the substrate for burst cycle $N_{b}=21$ at $V_{e p p}=643.4 \mathrm{~V}$. (c) The variation of peak-to-peak amplitude of the out-of-plane displacement response $\left(u_{y p p}\right)$ of the substrate over time $(t)$ and burst cycles $\left(N_{b}\right) .(d)$ Frequency spectra of the particle (solid line) and the substrate response (dashed line) to the SAW field at the excitation pulse amplitude of $V_{e p p}^{\max }=211.4 \mathrm{~V}$ at the burst cycle $N_{b}=9$ for particle $N_{p}=7$. Inset to Fig. 2.d: Transient time domain response of particle $N_{p}=7$. 
Figure 3. Trajectories of the microparticles in Sample Set A $(\boldsymbol{a})$ and B $(\boldsymbol{b})$ drifting on the soda-lime substrate when subjected to 21 successive burst cycles of SAW field. The location of each particle indicates its position on the substrate prior to the SAW exposure. The orientation of the acoustic wedge with respect to the sample is at $\alpha_{S A W}=10^{\circ}$ for the sample set A and $\alpha_{S A W}=0^{\circ}$ for Sample Set B. (c) Trajectories of particles $N_{p}=29$ (Group II) and $N_{p}=30$ of Sample Set A (Group I), (d) particle $N_{p}=26$ of Sample Set A (Group III), (e) $N_{p}=30$ of Sample Set B (Group I) and $(f) N_{p}=17$ of Sample Set B (Group I) when subjected to 21 successive burst cycles of SAW field. Each circled number indicates the location of the particle when it is subjected to the corresponding SAW burst cycles number $\left(N_{b}\right)$.

Figure 4. (a) The stop/motion pattern of the particles of Sample Set A during various burst cycles, categorized and sorted into three groups. (b) The percentage of the drifting particles on the substrate versus the excitation amplitude and burst cycle number (solid line) with a cubic polynomial curve fitted (dotted line). $(\boldsymbol{c})$ The CLA $\left(\theta_{l}^{c r}\right)$ versus the particle number $\left(N_{p}\right)$ in all MOIs categorized and sorted into three groups.

Figure 5. The CLA occurrence frequency $\left(f_{\theta_{t}^{c r}}\right)$ of the particles of Sample Set A. In this sample set, a total number of 29 particles out of a studied 30 particles started to drift under the influence of SAW field. 


\section{TABLE CAPTIONS}

Table 1: Summary of the CLA experiment results for the set of the particles of Sample Set A in all MOIs (1-5) with the average (AVG) and standard deviations (SD).

Table 2: Summary of the CLA experiment results for the microparticles of Sample Set A in MOI 1

categorized into three groups as Group I: $0.9^{\circ}<\theta_{l}^{c r}<1.2^{\circ}$, Group II: $2.0^{\circ}<\theta_{l}^{c r}<4.3^{\circ}$, Group III: $5.3^{\circ}<$ $\theta_{l}^{c r}<7.8^{\circ}$, with the average (AVG) and standard deviations (SD). 


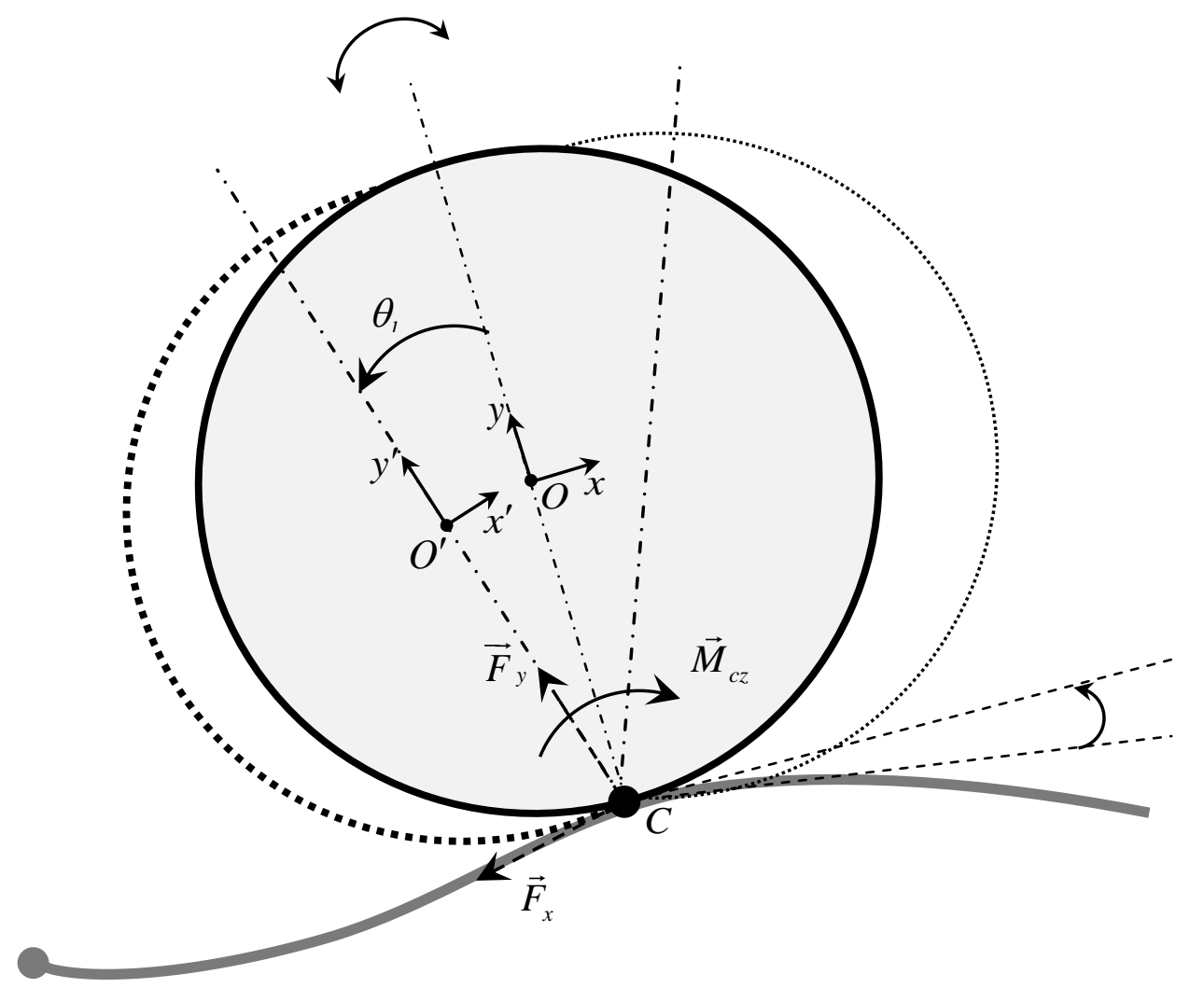

Figure 1.a 


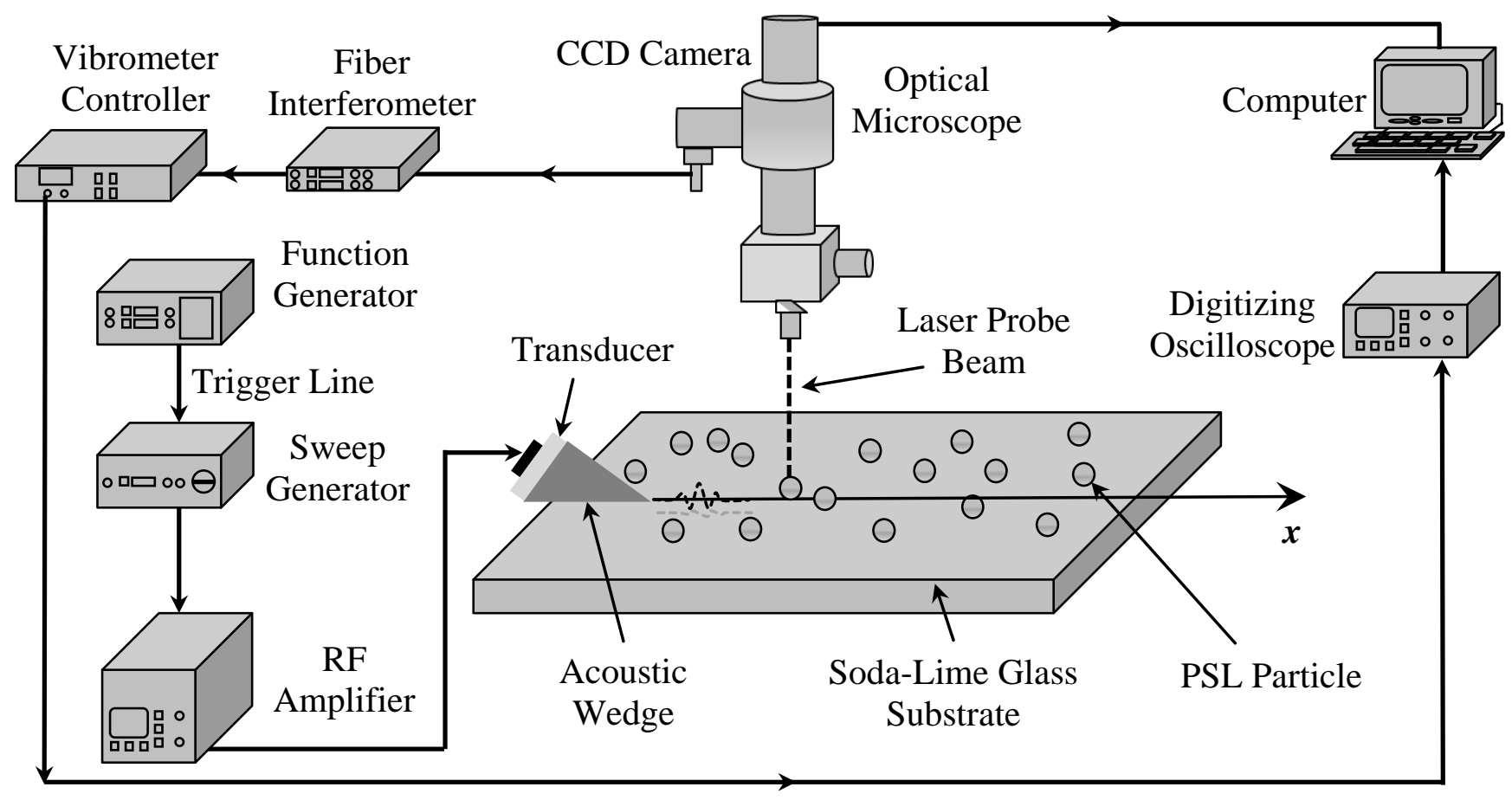

Figure 1.b 


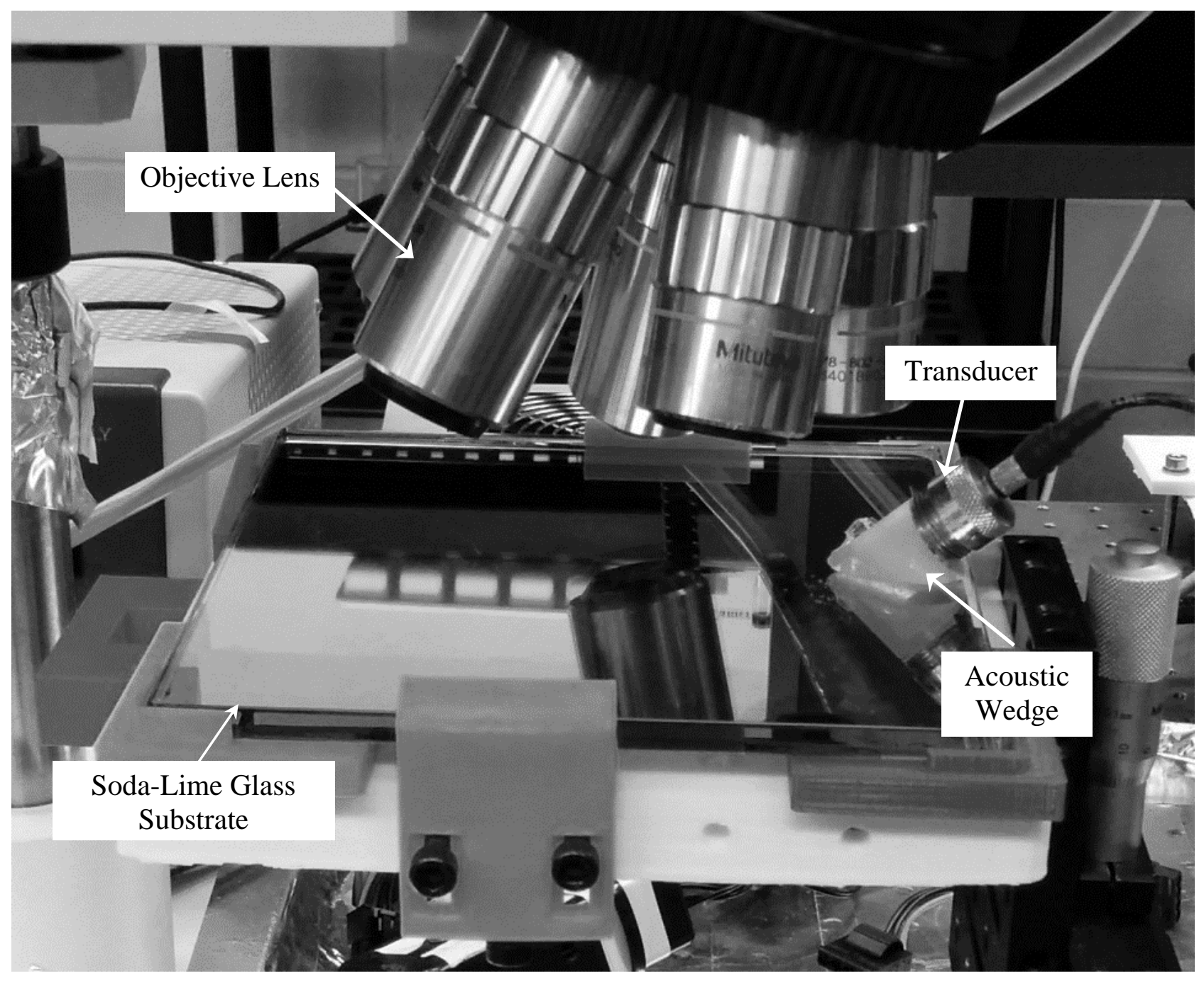

Figure 1.c 


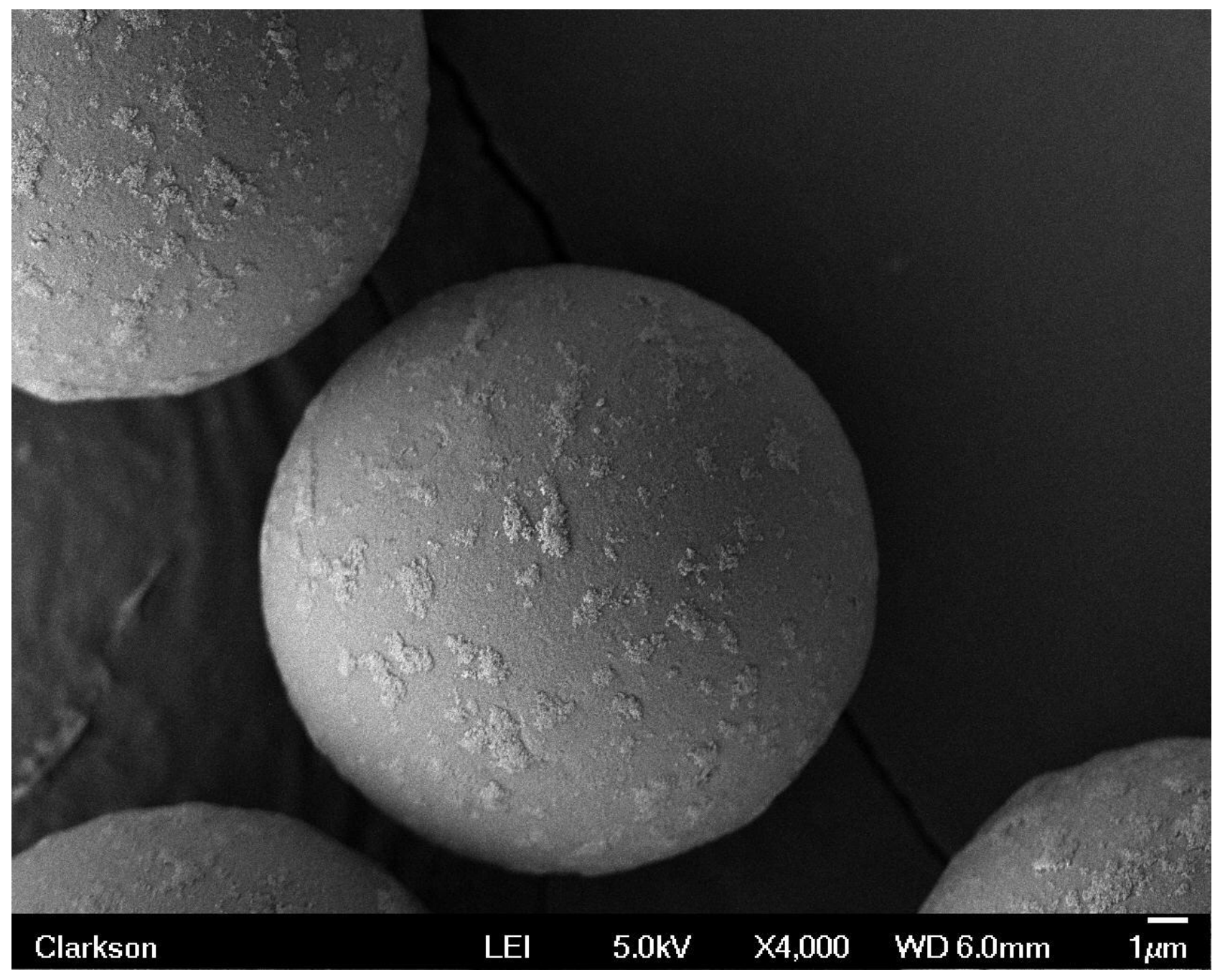

Figure 1.d.

Figure 1. (a) The leaning of a microparticle under the influence of a SAW field. (b) Instrumentation and connectivity diagram of the experimental set-up. Not to scale. (c) Close-up of the monitoring zone of the experimental set-up. (d) SEM image of microparticles utilized in the reported experiments, depicting surface morphology. 


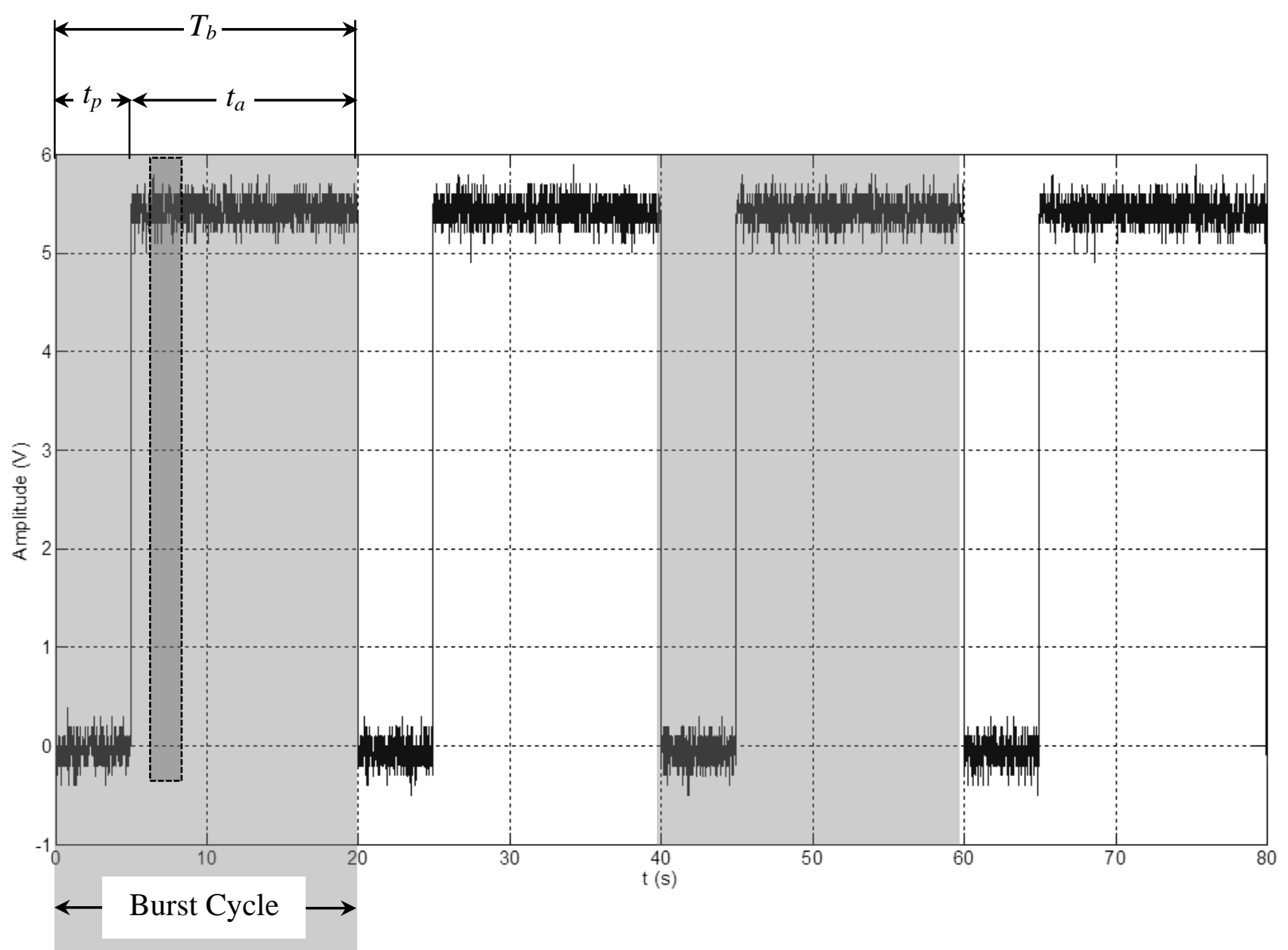

Figure 2.a 


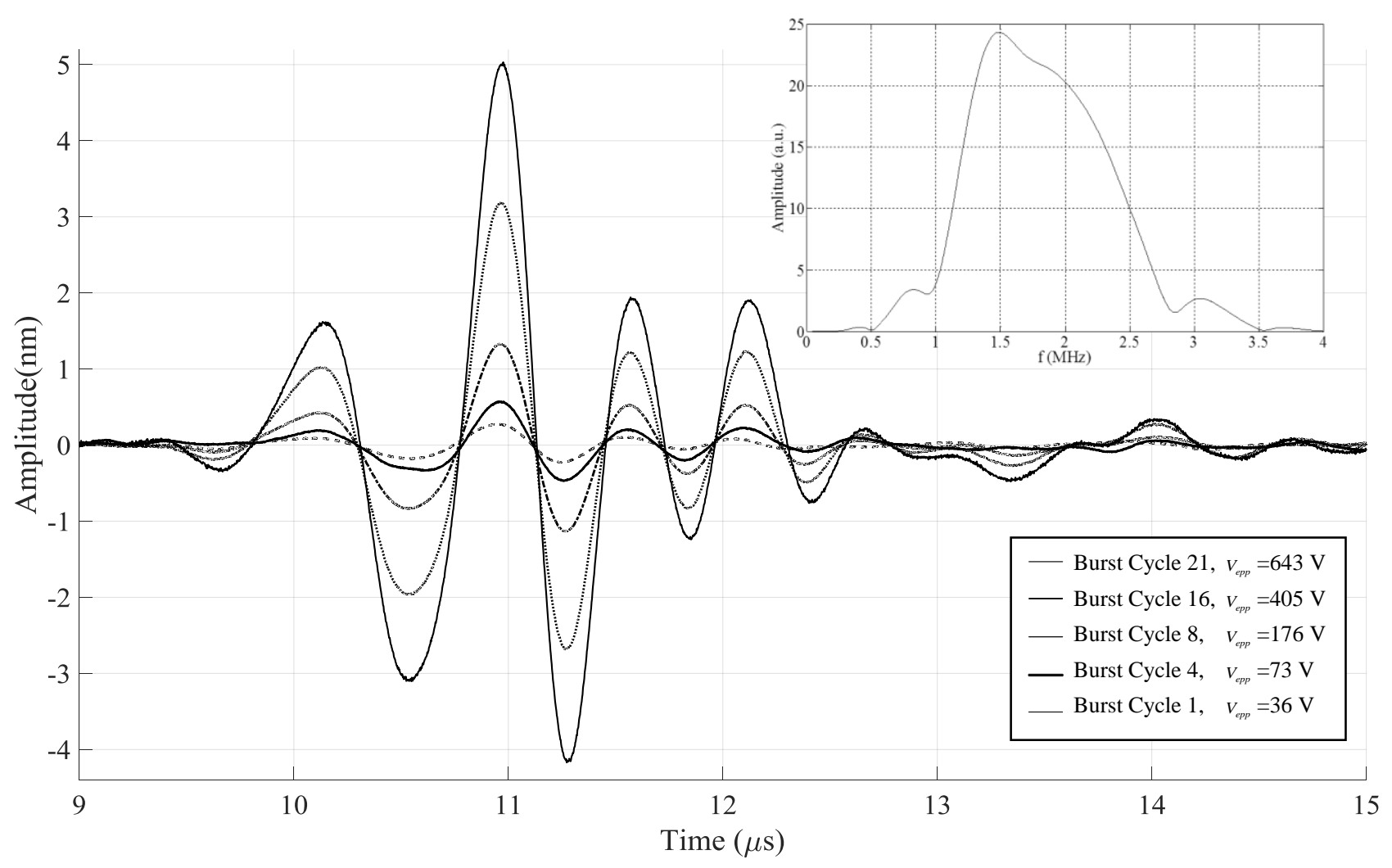

Figure 2.b 


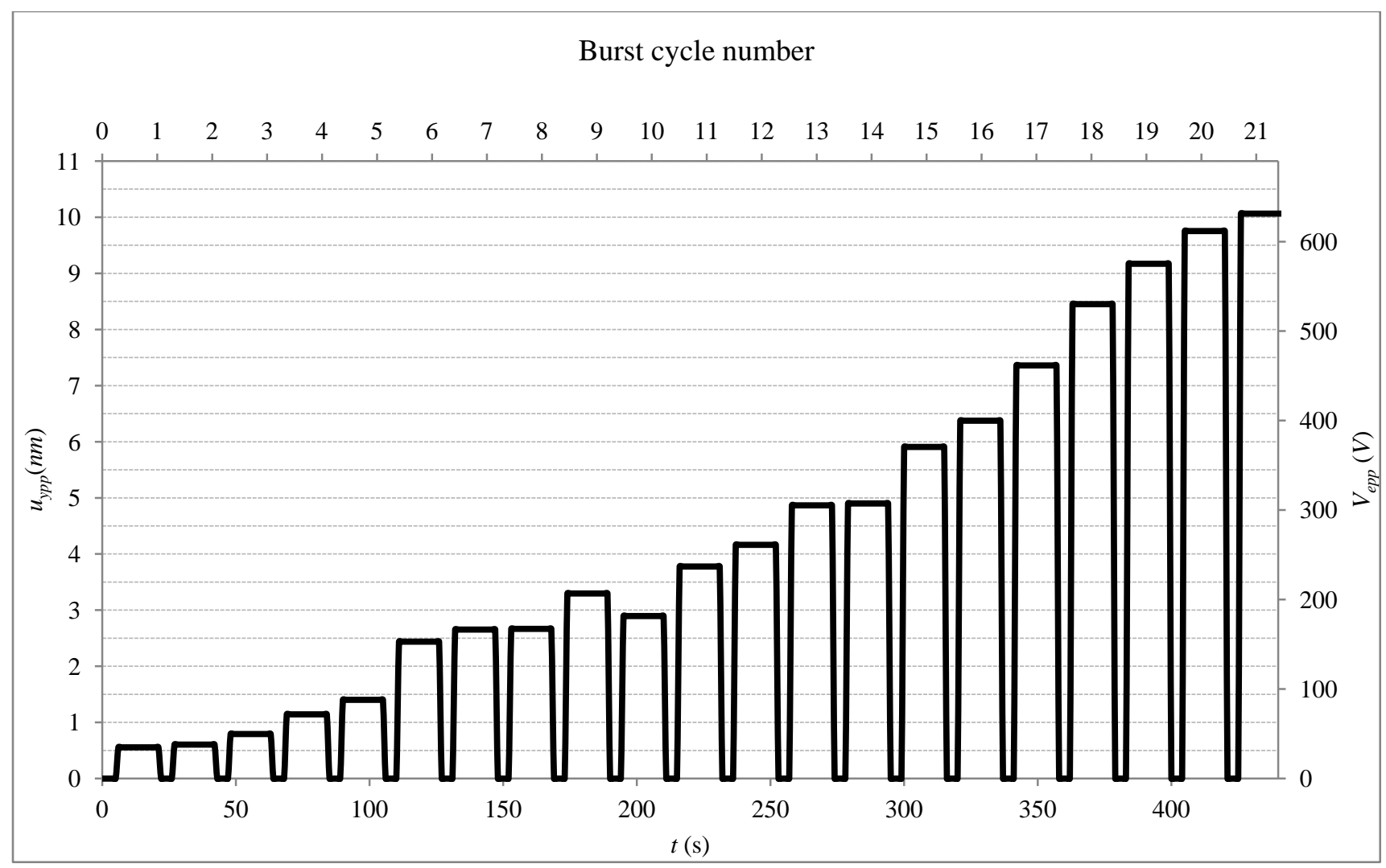

Figure 2.c. 


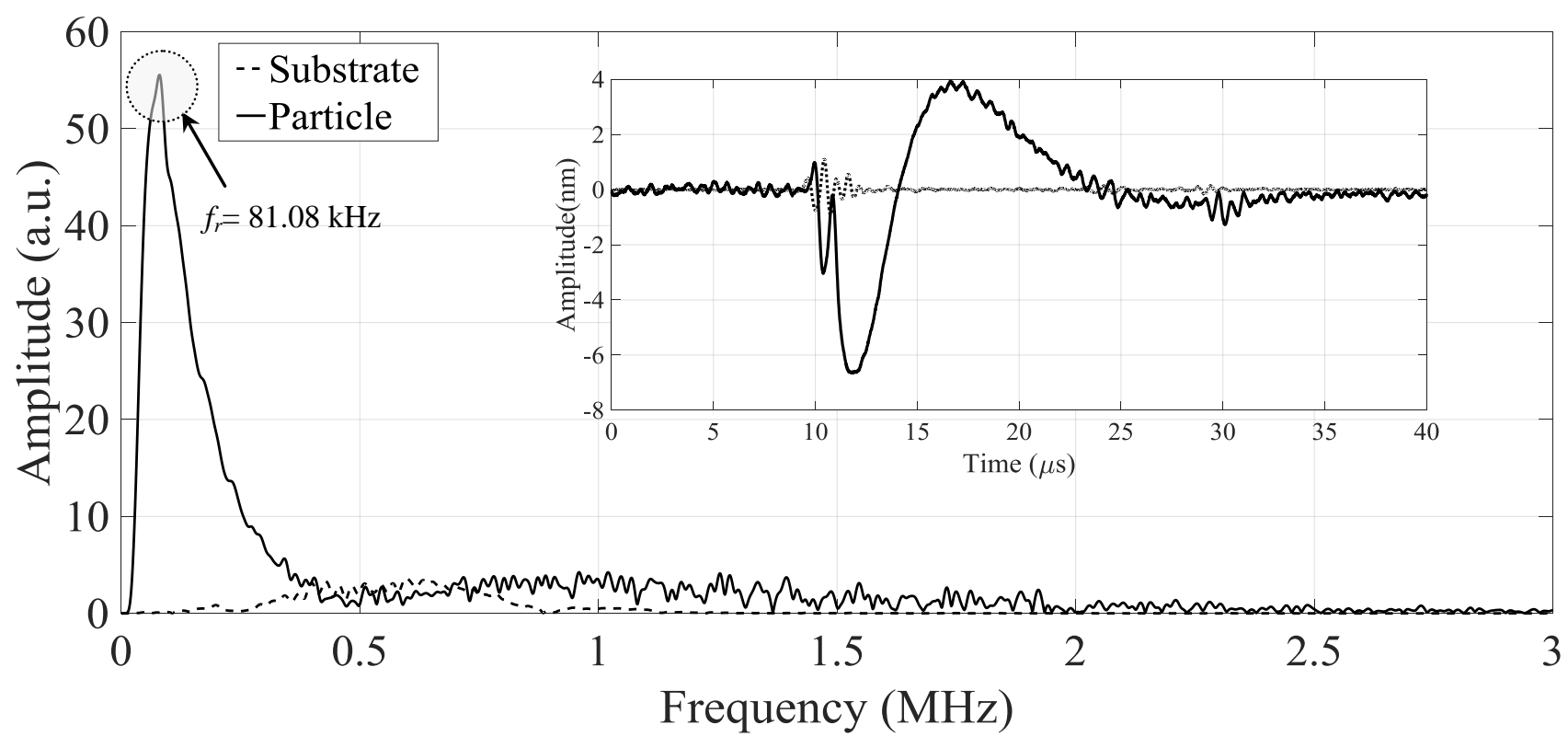

Figure 2.d

Figure 2. (a) A train of triggering burst cycles generated by the function generator. Each burst cycle consists of a train of triggering square pulses with a burst period of $T_{b}=20 \mathrm{sec}$ with a passive time duration of $t_{\mathrm{p}}=5 \mathrm{sec}$, active time duration of $t_{\mathrm{a}}=15 \mathrm{sec}$ and duty cycle of $\mathrm{D}=75 \%$. (b) Transient responses of the substrate $\left(u_{y}(t)\right)$ to excitation pulses for five specific burst cycles $\left(N_{b}=1\right.$, $4,8,16$ and 21) with the corresponding peak-to-peak excitation pulse voltages of $V_{e p p}=35.6,73.1$, 176.2, 405.2 and 643.4 V, respectively. Inset to Fig. 2.b: The spectral domain response of the substrate for burst cycle $N_{b}=21$ at $V_{e p p}=643.4 \mathrm{~V}$. (c) The variation of peak-to-peak amplitude of the out-of-plane displacement response $\left(u_{y p p}\right)$ of the substrate over time $(t)$ and burst cycles $\left(N_{b}\right) .(\boldsymbol{d})$ Frequency spectra of the particle (solid line) and the substrate response (dashed line) to the SAW field at the excitation pulse amplitude of $V_{e p p}^{\max }=211.4 \mathrm{~V}$ at the burst cycle $N_{b}=9$ for particle $N_{p}=7$. Inset to Fig. 2.d: Transient time domain response of particle $N_{p}=7$. 


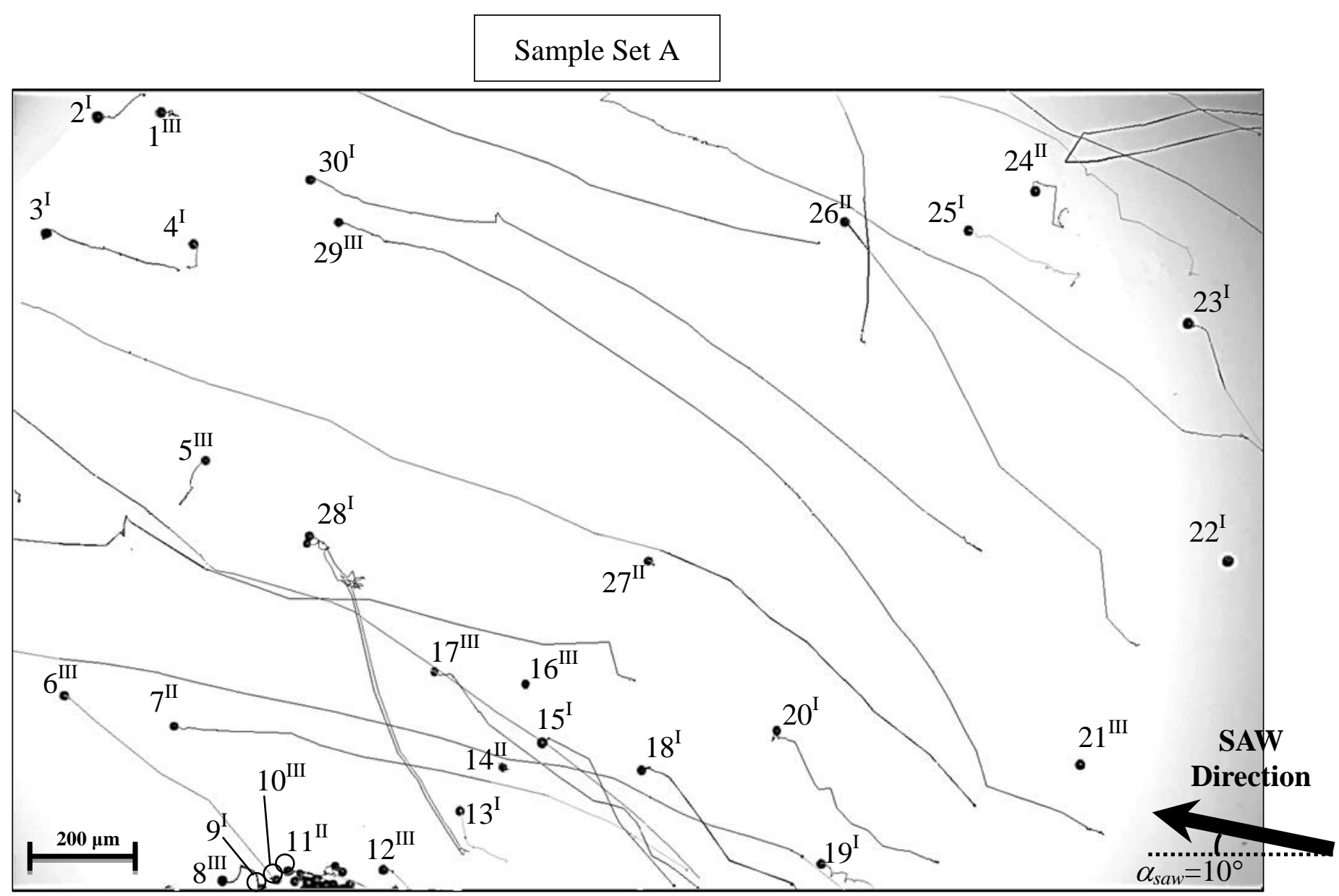

Figure 3.a 


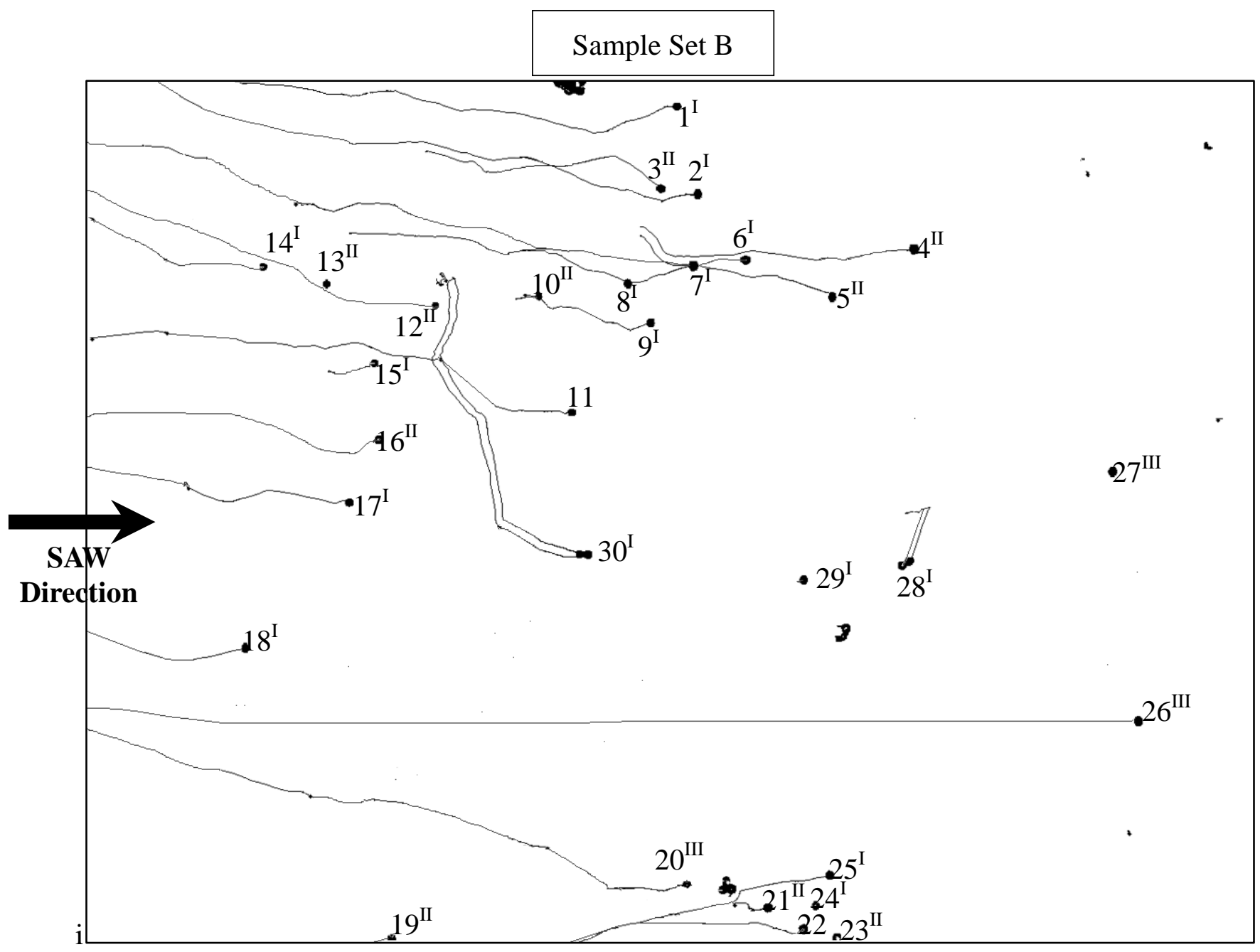

Figure 3.b 


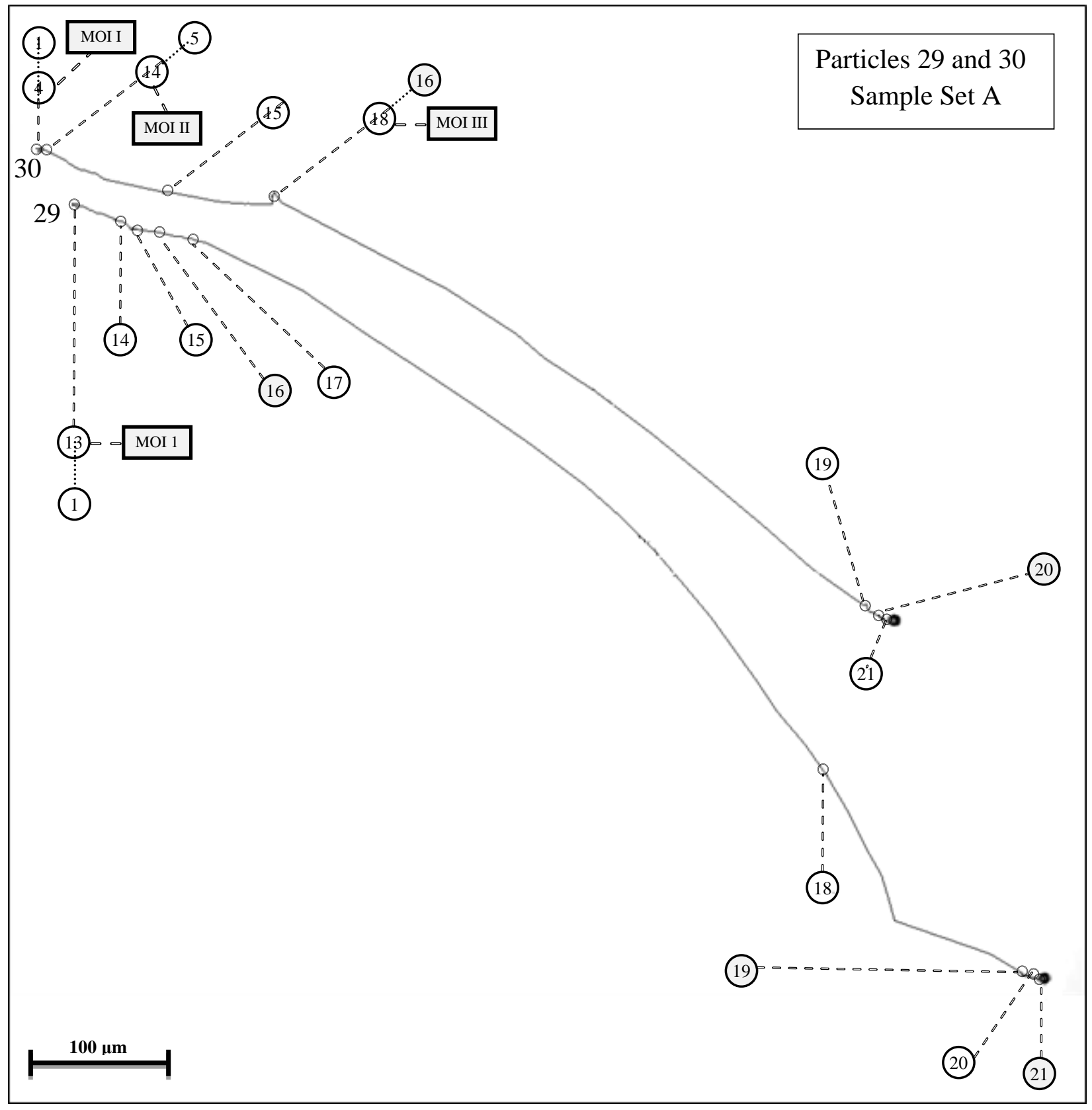

Figure 3.c 


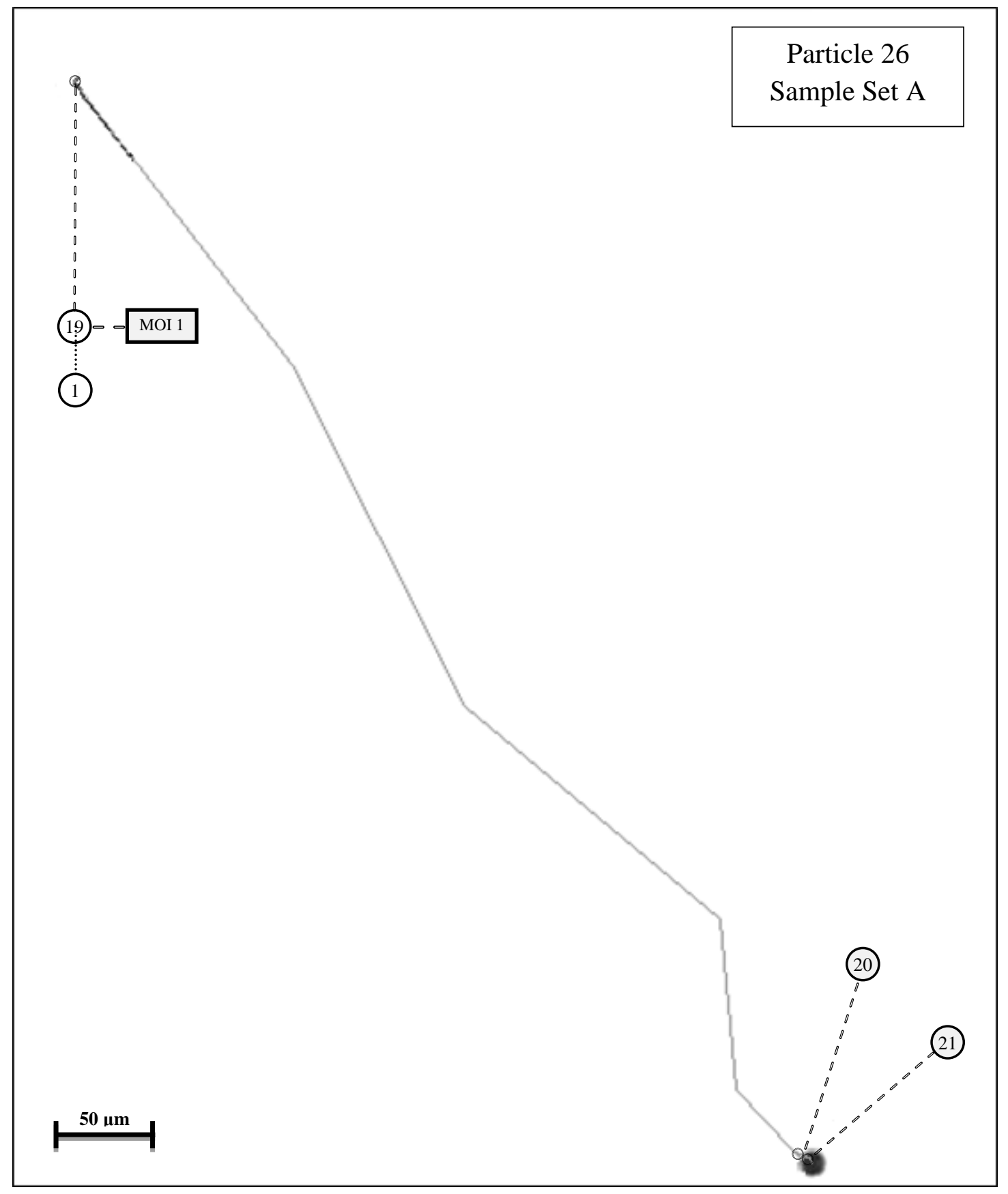

Figure 3.d 


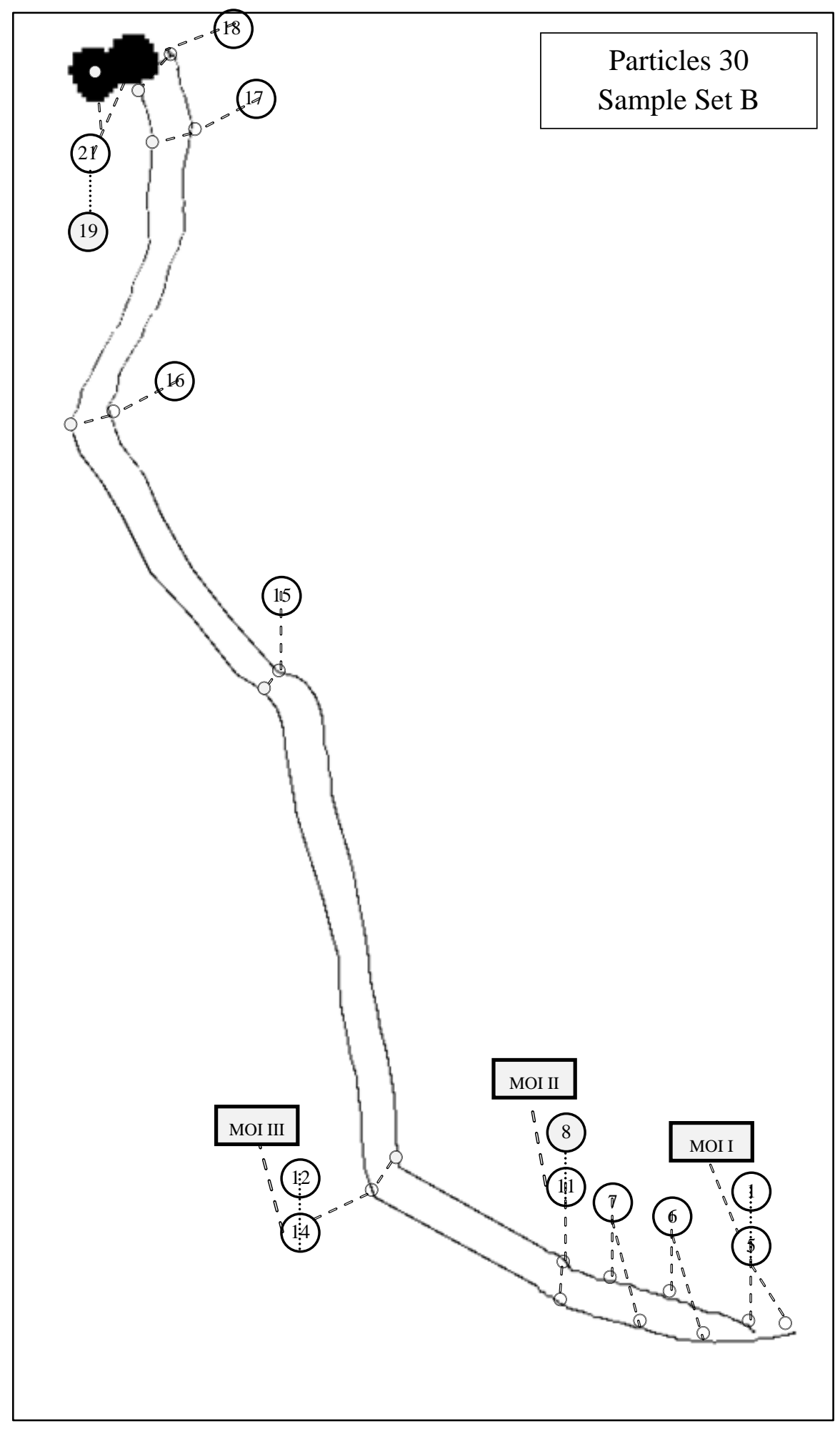

Fig. 3. e 


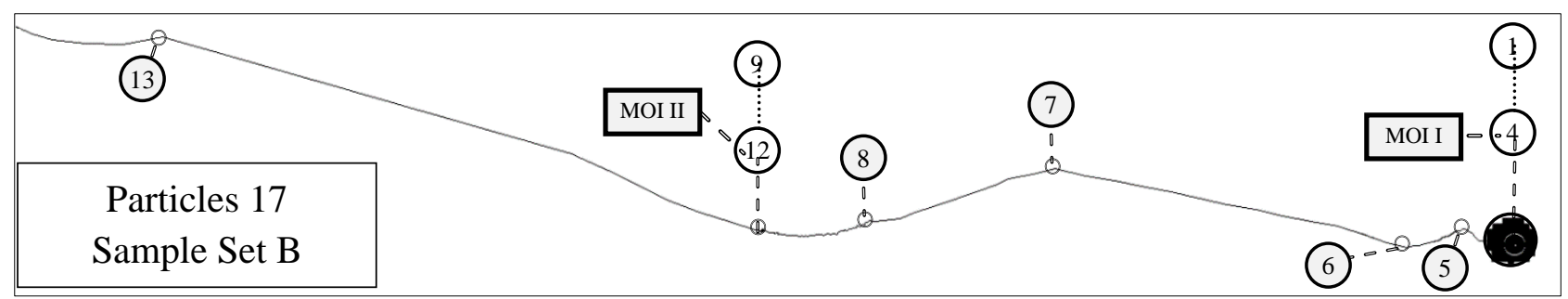

Fig. 3.f

Figure 3. Trajectories of the microparticles in Sample Set A (a) and B (b) drifting on the soda-lime substrate when subjected to 21 successive burst cycles of SAW field. The location of each particle indicates its position on the substrate prior to the SAW exposure. The orientation of the acoustic wedge with respect to the sample is at $\alpha_{S A W}=10^{\circ}$ for the sample set A and $\alpha_{S A W}=0^{\circ}$ for Sample Set B. (c) Trajectories of particles $N_{p}=29$ (Group II) and $N_{p}=30$ of Sample Set A (Group I), (d) particle $N_{p}=26$ of Sample Set A (Group III), (e) $N_{p}=30$ of Sample Set B (Group I) and $(\boldsymbol{f}) N_{p}=17$ of Sample Set B (Group I) when subjected to 21 successive burst cycles of SAW field. Each circled number indicates the location of the particle when it is subjected to the corresponding SAW burst cycles number $\left(N_{b}\right)$. 


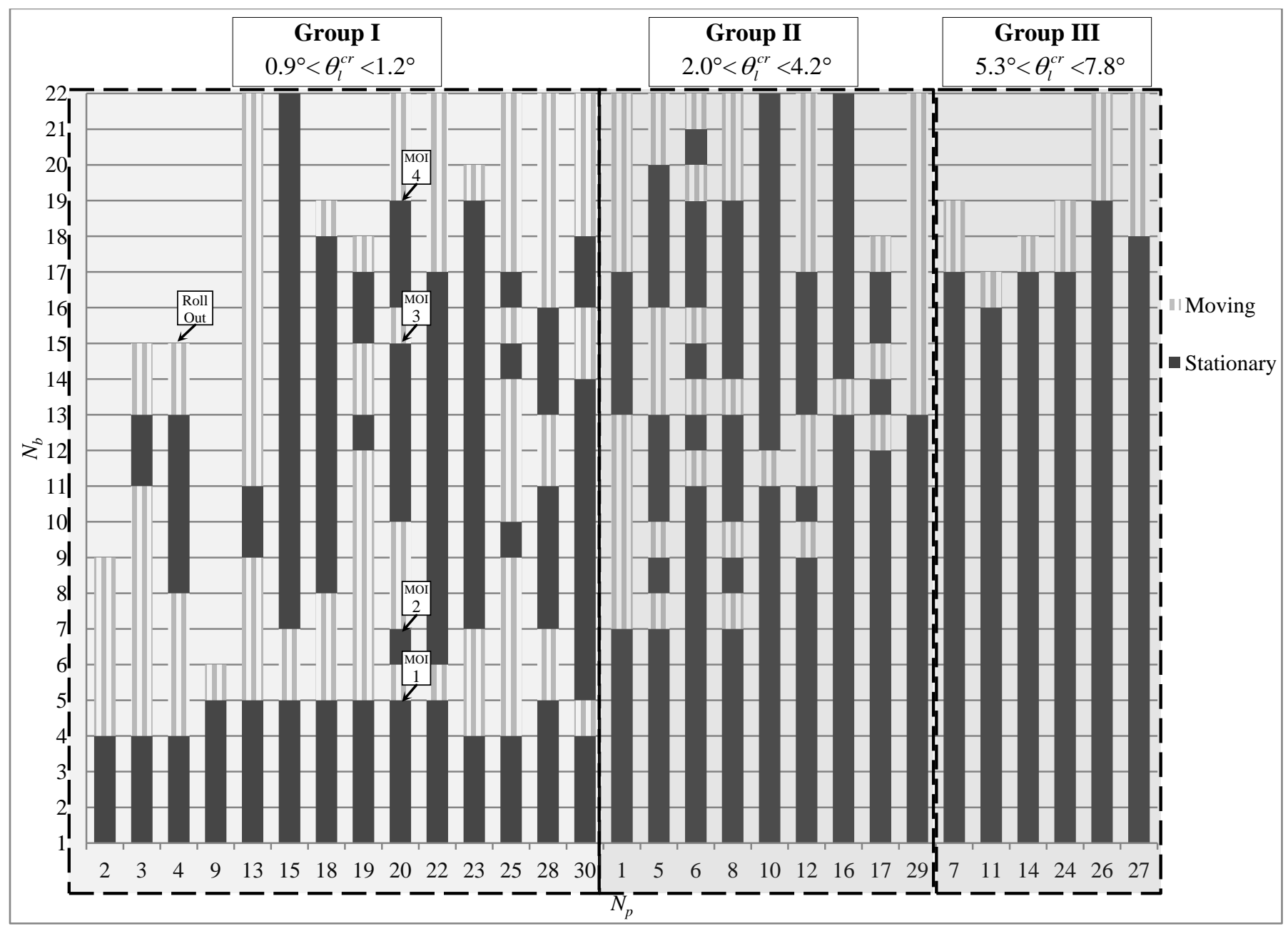

Figure 4.a 


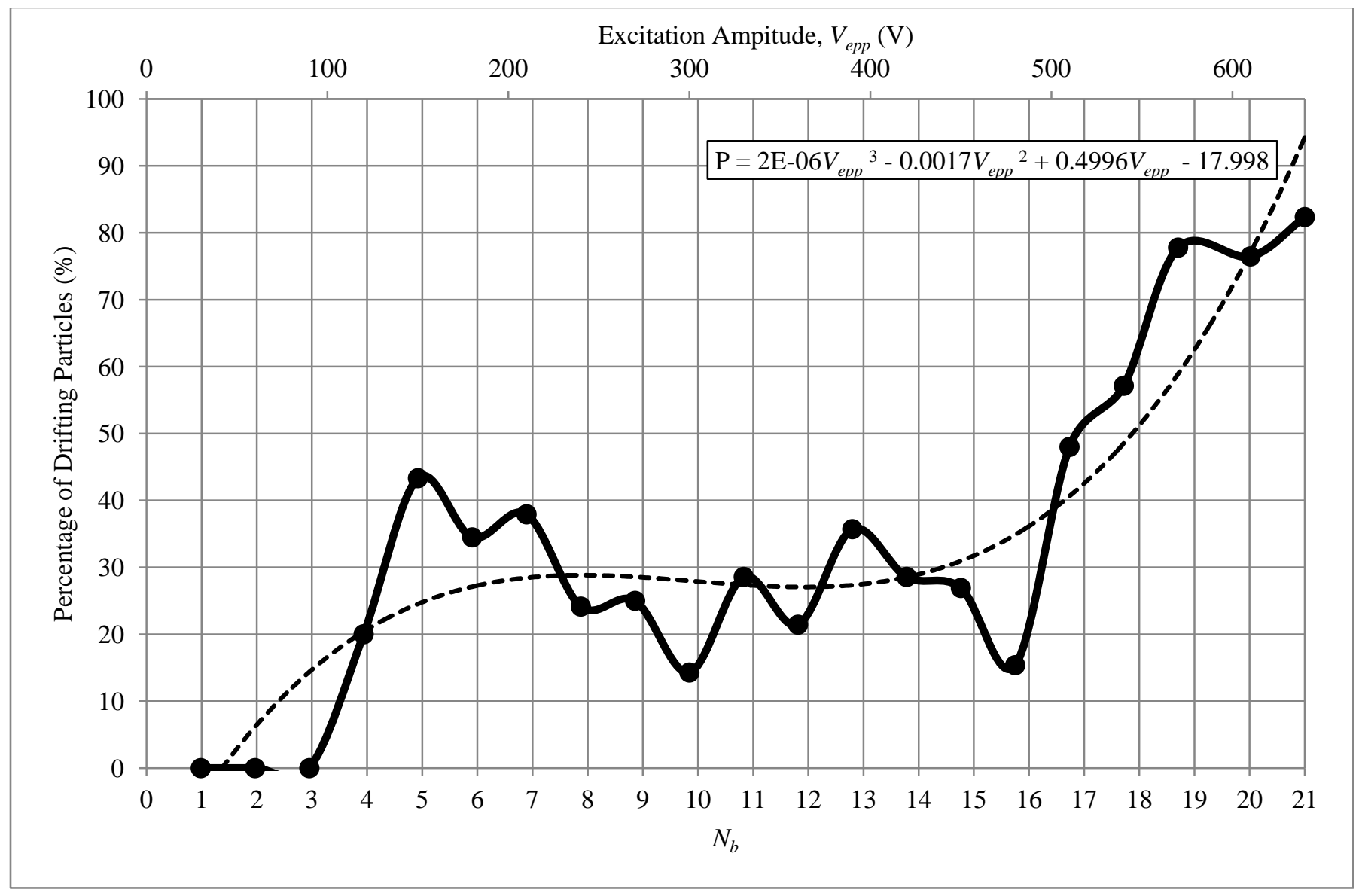

Figure 4.b 


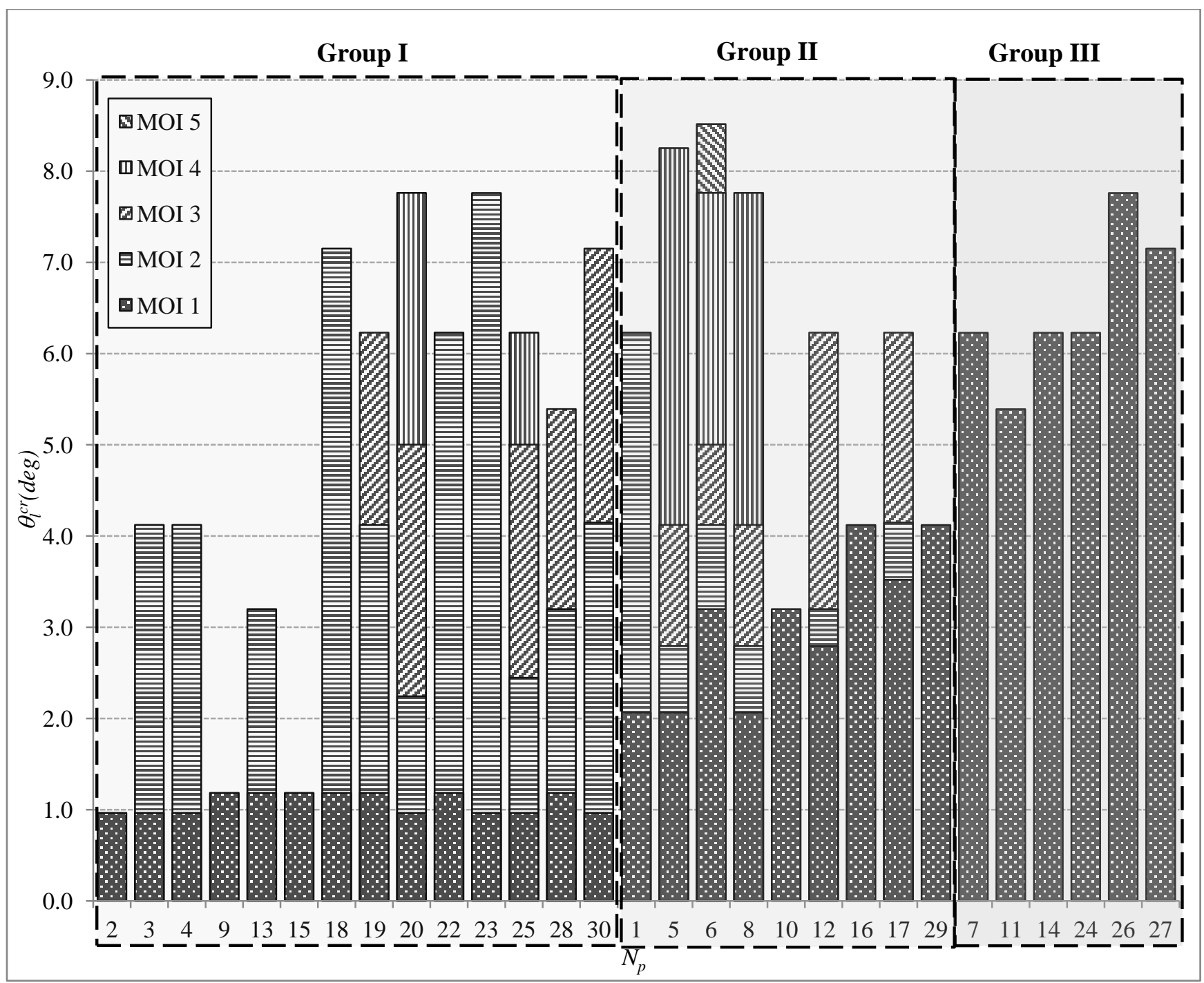

Figure 4.c

Figure 4. (a) The stop/motion pattern of the particles of Sample Set A during various burst cycles, categorized and sorted into three groups. (b) The percentage of the drifting particles on the substrate versus the excitation amplitude and burst cycle number (solid line) with a cubic polynomial curve fitted (dotted line). (c) The CLA ( $\left.\theta_{l}^{c r}\right)$ versus the particle number $\left(N_{p}\right)$ in all MOIs categorized and sorted into three groups. 


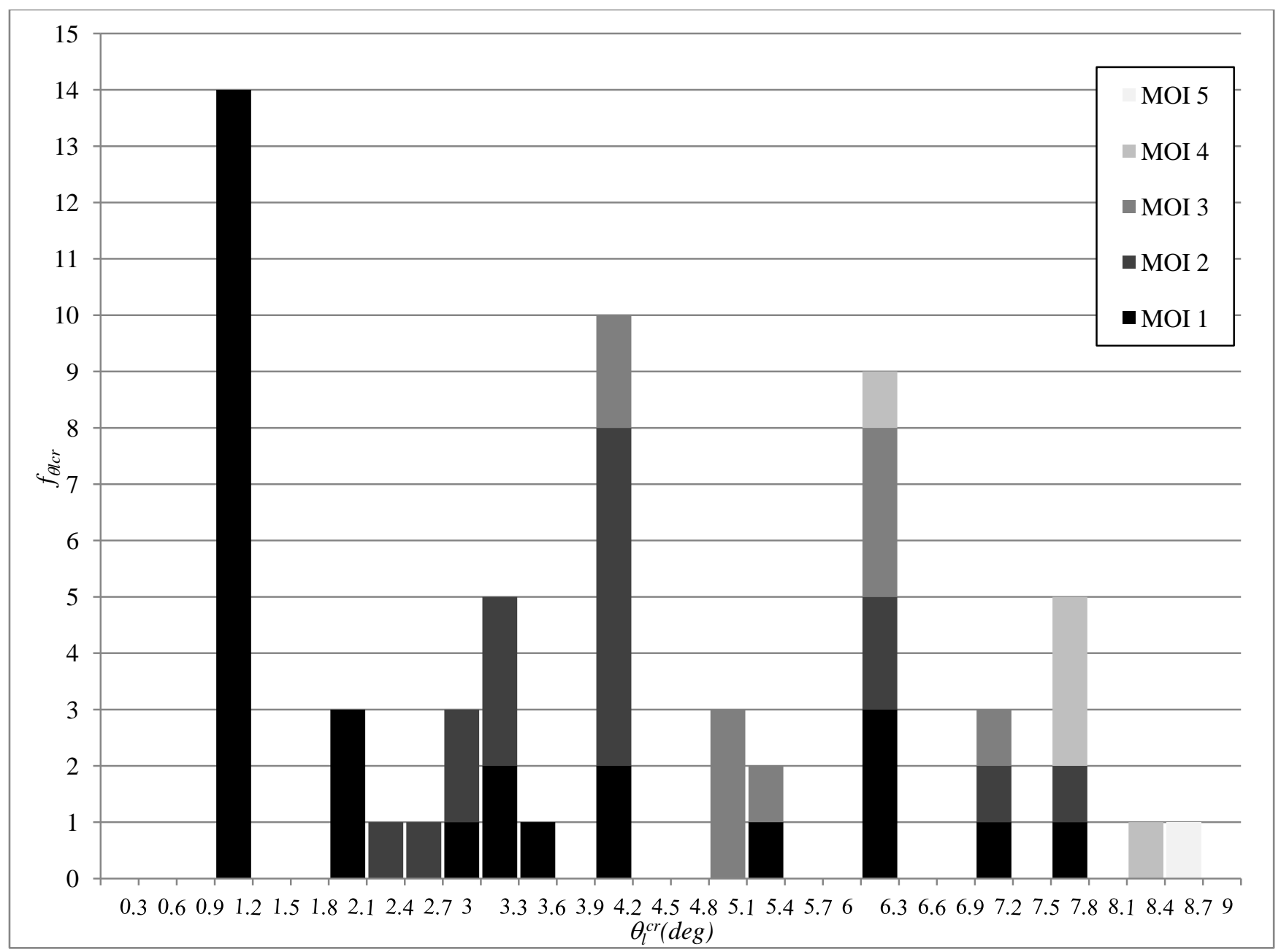

Figure 5.

Figure 5. The CLA occurrence frequency $\left(f_{\theta_{t}^{c r}}\right)$ of the particles of Sample Set A. In this sample set, a total number of 29 particles out of a studied 30 particles started to drift under the influence of SAW field. 
Table 1: Summary of the CLA experiment results for the set of the particles of Sample Set A in all MOIs (1-5) with the average (AVG) and standard deviations (SD).

\begin{tabular}{|c|c|c|c|c|c|c|c|c|c|c|c|c|c|c|c|}
\hline \multirow[b]{2}{*}{$N_{p}$} & \multicolumn{3}{|c|}{ MOI 1} & \multicolumn{3}{|c|}{ MOI 2} & \multicolumn{3}{|c|}{ MOI 3} & \multicolumn{3}{|c|}{ MOI 4} & \multicolumn{3}{|c|}{ MOI 5} \\
\hline & $N_{b}$ & $\begin{array}{c}u_{y p p}^{\max } \\
(n m)\end{array}$ & $\begin{array}{c}\theta_{l}^{c r} \\
(\operatorname{deg})\end{array}$ & $N_{b}$ & $\begin{array}{c}u_{y p p}^{\max } \\
(n m)\end{array}$ & $\begin{array}{c}\theta_{l}^{c r} \\
(d e g)\end{array}$ & $N_{b}$ & $\begin{array}{c}u_{y p p}^{\max } \\
(n m)\end{array}$ & $\begin{array}{c}\theta_{l}^{c r} \\
(d e g)\end{array}$ & $N_{b}$ & $\begin{array}{c}u_{y p p}^{\max } \\
(n m)\end{array}$ & $\begin{array}{c}\theta_{l}^{c r} \\
(d e g)\end{array}$ & $N_{b}$ & $\begin{array}{c}u_{y p p}^{\max } \\
(n m)\end{array}$ & $\begin{array}{c}\theta_{l}^{c r} \\
(d e g)\end{array}$ \\
\hline 1 & 7 & 2.44 & 2.06 & 17 & 7.36 & 6.23 & & & & & & & & & \\
\hline 2 & 4 & 1.14 & 0.96 & & & & & & & & & & & & \\
\hline 3 & 4 & 1.14 & 0.96 & 13 & 4.87 & 4.12 & & & & & & & & & \\
\hline 4 & 4 & 1.14 & 0.96 & 13 & 4.87 & 4.12 & & & & & & & & & \\
\hline 5 & 7 & 2.44 & 2.06 & 9 & 3.3 & 2.79 & 13 & 4.87 & 4.12 & 20 & 9.75 & 8.25 & & & \\
\hline 6 & 11 & 3.78 & 3.20 & 13 & 4.87 & 4.12 & 15 & 5.91 & 5.00 & 19 & 9.17 & 7.76 & 21 & 10.06 & 8.51 \\
\hline 7 & 17 & 7.36 & 6.23 & & & & & & & & & & & & \\
\hline 8 & 7 & 2.44 & 2.06 & 9 & 3.3 & 2.79 & 13 & 4.87 & 4.12 & 19 & 9.17 & 7.76 & & & \\
\hline 9 & 5 & 1.4 & 1.18 & & & & & & & & & & & & \\
\hline 10 & 11 & 3.78 & 3.20 & & & & & & & & & & & & \\
\hline 11 & 16 & 6.37 & 5.39 & & & & & & & & & & & & \\
\hline 12 & 9 & 3.3 & 2.79 & 11 & 3.78 & 3.20 & 17 & 7.36 & 6.23 & & & & & & \\
\hline 13 & 5 & 1.4 & 1.18 & 11 & 3.78 & 3.20 & & & & & & & & & \\
\hline 14 & 17 & 7.36 & 6.23 & & & & & & & & & & & & \\
\hline 15 & 5 & 1.4 & 1.18 & & & & & & & & & & & & \\
\hline 16 & 13 & 4.87 & 4.12 & & & & & & & & & & & & \\
\hline 17 & 12 & 4.16 & 3.52 & 14 & 4.9 & 4.15 & 17 & 7.36 & 6.23 & & & & & & \\
\hline 18 & 5 & 1.4 & 1.18 & 18 & 8.45 & 7.15 & & & & & & & & & \\
\hline 19 & 5 & 1.4 & 1.18 & 13 & 4.87 & 4.12 & 17 & 7.36 & 6.23 & & & & & & \\
\hline 20 & 5 & 1.14 & 0.96 & 7 & 2.65 & 2.24 & 15 & 5.91 & 5.00 & 19 & 9.17 & 7.76 & & & \\
\hline 21 & - & - & - & & & 6.23 & & & & & & & & & \\
\hline 22 & 5 & 1.4 & 1.18 & 17 & 7.36 & & & & & & & & & & \\
\hline 23 & 4 & 1.14 & 0.96 & 19 & 9.17 & 7.76 & & & & & & & & & \\
\hline 24 & 17 & 7.36 & 6.23 & & & & & & & & & & & & \\
\hline 25 & 4 & 1.14 & 0.96 & 10 & 2.89 & 2.45 & 15 & 5.91 & 5.00 & 17 & 7.36 & 6.23 & & & \\
\hline 26 & 19 & 9.17 & 7.76 & & & & & & & & & & & & \\
\hline 27 & 18 & 8.45 & 7.15 & & & & & & & & & & & & \\
\hline 28 & 5 & 1.4 & 1.18 & 11 & 3.78 & 3.20 & 16 & 6.37 & 5.39 & & & & & & \\
\hline 29 & 13 & 4.87 & 4.12 & & & & & & & & & & & & \\
\hline 30 & 4 & 1.14 & 0.96 & 14 & 4.9 & 4.15 & 18 & 8.45 & 7.15 & & & & & & \\
\hline AVG & 8.90 & 3.31 & 2.80 & 12.88 & 5.01 & 4.24 & 15.60 & 6.44 & 5.45 & 18.80 & 8.92 & 7.55 & 21 & 10.06 & 8.51 \\
\hline$\overline{\text { SD }}$ & 5.21 & 2.58 & 2.18 & 3.39 & 1.95 & 1.65 & 1.71 & 1.17 & 0.99 & 1.10 & 0.91 & 0.77 & - & - & - \\
\hline
\end{tabular}


Table 2: Summary of the CLA experiment results for the microparticles of Sample Set A in MOI 1 categorized into three groups as Group I: $0.9^{\circ}<\theta_{l}^{c r}<1.2^{\circ}$, Group II: $2.0^{\circ}<\theta_{l}^{c r}<4.3^{\circ}$, Group III: $5.3^{\circ}<$ $\theta_{l}^{c r}<7.8^{\circ}$, with the average (AVG) and standard deviations (SD).

\begin{tabular}{|c|c|c|c|c|c|c|c|c|c|c|c|}
\hline \multicolumn{4}{|c|}{ Group I } & \multicolumn{5}{|c|}{ Group II } & \multicolumn{4}{c|}{ Group III } \\
\hline$N_{p}$ & $N_{b}$ & $u_{y p p}^{\max }$ & $\theta_{l}^{c r}$ & $N_{p}$ & $N_{b}$ & $u_{y p p}^{\max }$ & $\theta_{l}^{c r}$ & $N_{p}$ & $N_{b}$ & $u_{y p p}^{\max }$ & $\theta_{l}^{c r}$ \\
\hline $\mathbf{2}$ & 4 & 1.14 & $\mathbf{0 . 9 6}$ & $\mathbf{1}$ & 7 & 2.44 & $\mathbf{2 . 0 6}$ & $\mathbf{7}$ & 17 & 7.36 & $\mathbf{6 . 2 3}$ \\
\hline $\mathbf{3}$ & 4 & 1.14 & $\mathbf{0 . 9 6}$ & $\mathbf{5}$ & 7 & 2.44 & $\mathbf{2 . 0 6}$ & $\mathbf{1 1}$ & 16 & 6.37 & $\mathbf{5 . 3 9}$ \\
\hline $\mathbf{4}$ & 4 & 1.14 & $\mathbf{0 . 9 6}$ & $\mathbf{6}$ & 11 & 3.78 & $\mathbf{3 . 2 0}$ & $\mathbf{1 4}$ & 17 & 7.36 & $\mathbf{6 . 2 3}$ \\
\hline $\mathbf{9}$ & 5 & 1.4 & $\mathbf{1 . 1 8}$ & $\mathbf{8}$ & 7 & 2.44 & $\mathbf{2 . 0 6}$ & $\mathbf{2 4}$ & 17 & 7.36 & $\mathbf{6 . 2 3}$ \\
\hline $\mathbf{1 3}$ & 5 & 1.4 & $\mathbf{1 . 1 8}$ & $\mathbf{1 0}$ & 11 & 3.78 & $\mathbf{3 . 2 0}$ & $\mathbf{2 6}$ & 19 & 9.17 & $\mathbf{7 . 7 6}$ \\
\hline $\mathbf{1 5}$ & 5 & 1.4 & $\mathbf{1 . 1 8}$ & $\mathbf{1 2}$ & 9 & 3.3 & $\mathbf{2 . 7 9}$ & $\mathbf{2 7}$ & 18 & 8.45 & $\mathbf{7 . 1 5}$ \\
\hline $\mathbf{1 8}$ & 5 & 1.4 & $\mathbf{1 . 1 8}$ & $\mathbf{1 6}$ & 13 & 4.87 & $\mathbf{4 . 1 2}$ & & & & \\
\hline $\mathbf{1 9}$ & 5 & 1.4 & $\mathbf{1 . 1 8}$ & $\mathbf{1 7}$ & 12 & 4.16 & $\mathbf{3 . 5 2}$ & & & & \\
\hline $\mathbf{2 0}$ & 5 & 1.14 & $\mathbf{0 . 9 6}$ & $\mathbf{2 9}$ & 13 & 4.87 & $\mathbf{4 . 1 2}$ & & & & \\
\hline $\mathbf{2 2}$ & 5 & 1.4 & $\mathbf{1 . 1 8}$ & & & & & & & & \\
\hline $\mathbf{2 3}$ & 4 & 1.14 & $\mathbf{0 . 9 6}$ & & & & & & & & \\
\hline $\mathbf{2 5}$ & 4 & 1.14 & $\mathbf{0 . 9 6}$ & & & & & & & & \\
\hline $\mathbf{2 8}$ & 5 & 1.4 & $\mathbf{1 . 1 8}$ & & & & & & & & \\
\hline $\mathbf{3 0}$ & 4 & 1.14 & $\mathbf{0 . 9 6}$ & & & & & & & & \\
\hline AVG & $\mathbf{4 . 5 7}$ & $\mathbf{1 . 2 7}$ & $\mathbf{1 . 0 7}$ & & $\mathbf{1 0 . 0 0}$ & $\mathbf{3 . 5 6}$ & $\mathbf{3 . 0 2}$ & & $\mathbf{1 7 . 3 3}$ & $\mathbf{7 . 6 8}$ & $\mathbf{6 . 5 0}$ \\
\hline SD & $\mathbf{0 . 5 1}$ & $\mathbf{0 . 1 3}$ & $\mathbf{0 . 1 1}$ & & $\mathbf{2 . 5 5}$ & $\mathbf{0 . 9 8}$ & $\mathbf{0 . 8 3}$ & & $\mathbf{1 . 0 3}$ & $\mathbf{0 . 9 8}$ & $\mathbf{0 . 8 3}$ \\
\hline $\mathbf{O c c u r r e n c e}$ & $\mathbf{4 7 \%}$ & $\mathbf{0 c c u r r e n c e}$ & $\mathbf{3 0 \%}$ & $\mathbf{O c c u r r e n c e}$ & & $\mathbf{2 0 \%}$ \\
\hline $\mathbf{R a t e}$ & & & & & & & & & \\
\hline
\end{tabular}

